\title{
ON COUPLED MULTIPARAMETER NONLINEAR ELLIPTIC SYSTEMS
}

\author{
BY \\ ROBERT STEPHEN CANTRELL
}

ABSTRACT. This paper considers the system of nonlinear Dirichlet boundary value problems

$$
\left\{\begin{array}{l}
L u(x)=\lambda f(u(x), v(x)) \\
L v(x)=\mu g(u(x), v(x))
\end{array}\right\}, \quad x \in \Omega
$$

a bounded domain in $\mathbf{R}^{n}$. Here $L$ is a strongly, uniformly elliptic linear partial differential operator, $\lambda, \mu$ are real parameters, and $f, g: \mathbf{R}^{2} \rightarrow R$ are smooth with

$$
f(0,0)=0=g(0,0) .
$$

A detailed analysis of the solution set to the system is given from the point of view of several parameter bifurcation theory.

1. Introduction. Much attention has been focused recently on several parameter bifurcation problems (see, for example, Alexander and Antman [3, 4], Cantrell [7] and the references therein). One of the principal mathematical applications of the theory (which the above-mentioned and other works have developed) is to the bifurcation phenomena of coupled systems of nonlinear elliptic boundary value problems depending on several parameters. The interest in such problems is strong. For instance, the significance of componentwise positive solutions to such systems in the applications has been noted in the recent survey article of Lions [21]. Also, Protter [22] notes that connection of the study of the linearized version of such systems to the problem of determining lower bounds on eigenvalues of higher-order differential operators.

One natural way to classify such systems is according to the manner in which they are coupled. The terms strongly coupled and weakly coupled have more or less standard usage in the literature. However, neither term conveys the distinction we seek to make. Therefore we have chosen to adopt the terms state coupled and parametrically coupled in this paper. We shall say that a system of nonlinear elliptic boundary value problems is state coupled if the linearization about the trivial solution (we assume tacitly that such exists) of the system is coupled in the state variables. It is said to be parametrically coupled if the linearization is coupled only by the parameters. We note that the term completely coupled used by Zachmann $[26]$ is essentially the same as our term state coupled.

Received by the editors March 7, 1985.

1980 Mathematics Subject Classification. Primary 58E07; Secondary 35J65.

(c) 1986 American Mathematical Society $0002-9947 / 86 \$ 1.00+\$ .25$ per page 
Let us now illustrate these terms. Consider

$$
\left\{\begin{aligned}
&\left(p_{i}\left(t_{i}\right) y_{i}^{\prime}\left(t_{i}\right)\right)^{\prime}+\left\{q_{i}\left(t_{i}\right)\right.\left.+\sum_{j=1}^{k} \lambda_{j} a_{i j}\left(t_{i}\right)\right\} y_{i}\left(t_{i}\right) \\
& \quad+r_{i}\left(t_{i}, \lambda_{1}, \ldots, \lambda_{k}, y_{i}\left(t_{i}\right), y_{i}^{\prime}\left(t_{i}\right)\right)=0 \\
& \\
& \alpha_{i} y_{i}\left(A_{i}\right)+\alpha_{i}^{\prime} y_{i}^{\prime}\left(A_{i}\right)=0 \\
& \beta_{i} y_{i}\left(B_{i}\right)+\beta_{i}^{\prime} y_{i}^{\prime}\left(B_{i}\right)=0 \\
& t_{i} \in\left[A_{i}, B_{i}\right], \quad\left(\left|\alpha_{i}\right|+\left|\alpha_{i}^{\prime}\right|\right)\left(\left|\beta_{i}\right|+\left|\beta_{i}^{\prime}\right|\right)>0, \quad i=1,2, \ldots, k .
\end{aligned}\right.
$$

If $r_{i}:\left[A_{i}, B_{i}\right] \times \mathbf{R}^{k} \times \mathbf{R}^{2} \rightarrow \mathbf{R}$ is such that $r_{i}\left(t, \lambda_{1}, \ldots, \lambda_{k}, w, z\right)=o(|w|+|z|)$ uniformly for $\left(t, \lambda_{1}, \ldots, \lambda_{k}\right)$ in compact subsets of $\left[A_{i}, B_{i}\right] \times \mathbf{R}^{k}$ for $i=1, \ldots, k$, then (1.1) is an example of a parametrically coupled system. If $k=1,(1.1)$ is a problem whose bifurcation phenomena were described by Crandall and Rabinowitz [10], Rabinowitz [24], Turner [25], and others as an application of the odd multiplicity bifurcation theorem of Krasnosel'skii $[\mathbf{2 0}]$. It is also intimately related to the development of the now famous global extension of Krasnosel'skii's theorem, due to Rabinowitz [24].

If $k>1,(1.1)$ is a nonlinear perturbation of the Klein Oscillation Problem $[\mathbf{1 3}, \mathbf{1 9}]$. In this case, (1.1) was first studied by Browne and Sleeman $[\mathbf{5}, \mathbf{6}]$. They demonstrated, under suitable conditions on the coefficient functions, the existence of unbounded branches of solutions (having a specified nodal structure in each state component) to (1.1) emanating from the countably infinite set of points $\left(\lambda_{1}, \ldots, \lambda_{k}\right)$ in $\mathbf{R}^{k}$ for which the associated Klein Oscillation Problem possesses a solution $\left(u_{1}, \ldots, u_{k}\right)$ with $u_{i}$ nontrivial for $i=1,2, \ldots, k$. Later, Cantrell $[\mathbf{8}, \mathbf{9}]$, using methods analogous to those of $[\mathbf{2 0}$ and $\mathbf{2 4}]$, showed that the set of bifurcation points for (1.1) is actually the union of $k$ sets, each of which is a countably infinite collection of pairwise disjoint unbounded $k-1$ analytic manifolds in $\mathbf{R}^{k}$. The bifurcation points of Browne and Sleeman $[\mathbf{5}, \mathbf{6}]$ are the intersections of $k$ of the $k-1$ analytic manifolds, one from each of the $k$-collections. Furthermore, it is also shown in $[\mathbf{8}$ and $\mathbf{9}]$ that the nature of the nontrivial solutions near a bifurcation point is intimately related to the number (ranging from 1 to $k$ ) of the afore-mentioned $k-1$ manifolds upon which the point lies.

An example of a state coupled system of nonlinear boundary value problems is given by

$$
\left\{\begin{aligned}
&-\left(p u^{\prime}\right)^{\prime}+P u+r v=\lambda_{1} u+\lambda_{1} N_{11}(u, v)+\lambda_{2} N_{21}(u, v), \\
&-\left(q v^{\prime}\right)^{\prime}+Q v+r u=\lambda_{2} v+\lambda_{2} N_{12}(u, v)+\lambda_{2} N_{22}(u, v), \\
& \alpha_{11} u(0)+\alpha_{12} u^{\prime}(0)=0=\alpha_{21} v(0)+\alpha_{22} v^{\prime}(0), \\
& \beta_{11} u(1)+\beta_{12} u^{\prime}(1)=0=\beta_{21} v(1)+\beta_{22} v^{\prime}(1) .
\end{aligned}\right.
$$

(Here we assume that $N_{i j}$ is higher order in $|u|+|v|$ for $i, j=1,2$.) This system was studied by Zachman [26]. He utilized a modified Lyapunov-Schmidt reduction and the Weierstrass Preparation Theorem to establish that if $\left(\lambda_{1}, \lambda_{2}\right)$ is near a simple "eigenvalue" of the linearization of (1.2), then the number of small solutions of (1.2) corresponds to the number of real roots of a certain associated polynomial.

This paper considers the solution set of another class of state coupled multiparameter systems of nonlinear boundary value problems. The most general form of 
such systems is as follows:

$$
\left\{\begin{aligned}
A u_{i}(x) & =\lambda_{i} f_{i}\left(u_{1}(x), u_{2}(x), \ldots, u_{n}(x)\right), \quad x \in \Omega, \\
u_{i}(x) & \equiv 0, \quad i=1, \ldots, n, x \in \partial \Omega,
\end{aligned}\right.
$$

where $\Omega$ is a bounded domain in $\mathbf{R}^{k}, k \geq 1$, with sufficiently smooth boundary and $\lambda=\left(\lambda_{1}, \ldots, \lambda_{n}\right) \in \mathbf{R}^{n}$. $\mathcal{A}$ is a uniformly elliptic linear or quasilinear differential operator and $f_{i}: \mathbf{R}^{n} \rightarrow \mathbf{R}$ is $C^{\infty}$ and satisfies $f_{i}(0,0, \ldots, 0)=0$ for $i=1,2, \ldots, n$.

This last assumption on $f_{i}$ insures that $u_{1}=u_{2}=\cdots=u_{n} \equiv 0$ is a solution to (1.3) for any value of the multiparameter $\lambda$. Thus, as with the preceding examples, bifurcation theory provides an appropriate framework for an analysis of (1.3). In particular, if $\mathcal{G}=A^{-1}$ exists, then (1.3) may be equivalently expressed by an equation of the form

$$
e=A(\lambda) e+H(\lambda, e),
$$

where $\lambda=\left(\lambda_{1}, \ldots, \lambda_{n}\right) \in \mathbf{R}^{n}$ and $e \in E$, a real Banach space. Here $A: \mathbf{R}^{n} \rightarrow$ $K(E)$ (the Banach space of compact linear operators on $E$ ) is continuous and $H: \mathbf{R}^{n} \times E \rightarrow E$ is completely continuous and higher-order in $e$ (uniformly for $\lambda$ contained in compact subsets of $\left.\mathbf{R}^{n}\right) . A(\cdot)$ is also positive homogeneous of degree one, i.e.

$$
A(t \lambda)=t A(\lambda)
$$

for $t>0$ and $\lambda \in \mathbf{R}^{n}$. This last fact allows us to invoke the odd multiplicity bifurcation results of [3 and $\mathbf{7}]$ in the case of (1.3).

Our attention will primarily be on the case $n=2$, i.e. the system

$$
A u(x)=\lambda f(u(x), v(x)), \quad A v(x)=\mu g(u(x), v(x)),
$$

$x \in \Omega, u(x) \equiv 0 \equiv v(x), x \in \partial \Omega$. The main purpose of this paper is to provide a somewhat detailed description of the bifurcation phenomena associated with (1.6). To this end, we utilize several different methods of the general theory. Each method allows us to establish a different qualitative or quantitative aspect of the bifurcation phenomena. In combination, an enhanced description results.

We begin in $\S 2$ with an examination of the linearization of $(1.6)$, which has the form

$$
L u=\lambda f_{1} u+\lambda f_{2} v, \quad L v=\mu g_{1} u+\mu g_{2} v,
$$

where $L$ is an invertible strongly elliptic linear operator on $\Omega$,

$$
f_{1}=\left.\frac{\partial f}{\partial x}\right|_{(0,0)}, \quad f_{2}=\left.\frac{\partial f}{\partial y}\right|_{(0,0)}, \quad g_{1}=\left.\frac{\partial g}{\partial x}\right|_{(0,0)}, \quad g_{2}=\left.\frac{\partial g}{\partial y}\right|_{(0,0)} .
$$

As is well known, values of $(\lambda, \mu)$ for which (1.6) exhibits branching behavior at $(\lambda, \mu, 0,0)$ must be such that $(1.7)$ has a nontrivial solution $(u, v)$ at $(\lambda, \mu)$. We explicitly calculate this set (which we denote $\Sigma_{A}$ ). We also determine the values of $(\lambda, \mu)$ for which the operator

$$
I-A(\lambda, \mu)
$$

where

$$
A(\lambda, \mu)=\left[\begin{array}{ll}
\lambda f_{1} L^{-1} & \lambda f_{2} L^{-1} \\
\mu g_{1} L^{-1} & \mu g_{2} L^{-1}
\end{array}\right]
$$


has generalized null space of dimension $>1$ (i.e. algebraic multiplicity $>1$ ). This information will be crucial in determining (both locally and globally) the bifurcation phenomena associated with (1.6).

Certain local aspects of these phenomena are discussed, beginning in $\S 3$. We employ a Lyapunov-Schmidt method along the lines of Zachmann [26] in the situation of (1.3), thereby determining conditions under which one may obtain the number of "small" solutions for parameter values near an algebraically simple element of $\Sigma_{A}$. Furthermore, in the spirit of Krasnosel'skii-Rabinowitz [20, 24], we characterize components of nontrivial solutions emanating from the "first curves" of $\Sigma_{A}$ as being of one sign (at least locally). We also observe that, in case $A$ is a Sturm-Liouville operator, this phenomenon has natural analogues for the "higher curves" of $\Sigma_{A}$.

In $\S 4$, we note the existence of certain sets of nontrivial bifurcating solutions with topological dimension at least 2 at every point. Since (1.6) (or more generally (1.3)) is equivalent to a system (1.4) in which condition (1.5) holds, the global multidimensional bifurcation theory of Alexander and Antman [3] is applicable for this purpose.

We give two main global results. In $\S 5$, we show that provided

$$
\frac{\sqrt{f_{1} g_{2}}-\sqrt{f_{2} g_{1}}}{\sqrt{f_{1} g_{2}}+\sqrt{f_{2} g_{1}}} \geq \frac{\lambda_{1}}{\lambda_{2}}
$$

where $\lambda_{1}$ and $\lambda_{2}$ are the first two eigenvalues of $L$ (see (1.7)), the continuum emanating from an algebraically simple element $\left(\lambda_{0}, \mu_{0}\right)$ of $\Sigma_{A}$ is unbounded in directions transverse to $\Sigma_{A}$ at $\left(\lambda_{0}, \mu_{0}\right)$. This result relies heavily on our analysis in $\S 2$ of the linear system (1.7). In $\S 6$, we place some additional conditions on the maps $f$ and $g$. These conditions, along with our detailed understanding of (1.7), allow us to use maximum principle arguments to assert global persistence of solutions with positive $u$ and $v$ components.

As noted, we treat only the case $n=2$ in $\S \S 2-6$. However, many of the methods and results of these sections have analogues in the case $n>2$. We make the relevant observations in $\S 7$. Finally, in $\S 8$, we conclude the article by illustrating our techniques and results with a detailed examination of a particular system of Sturm-Liouville boundary value problems.

2. The linearized system. Consider (1.7). Assume that $L$ has eigenvalues $0<\lambda_{1}<\lambda_{2}<\lambda_{3}<\cdots \rightarrow+\infty$. If $u=\alpha x_{n}$ and $v=\beta x_{n}$, where $x_{n}$ is an eigenfunction corresponding to the $n$th eigenvalue $\lambda_{n}$ of $L,(1.7)$ becomes

$$
\left(\begin{array}{cc}
\lambda_{n}-\lambda f_{1} & -\lambda f_{2} \\
-\mu g_{1} & \lambda_{n}-\mu g_{2}
\end{array}\right)\left(\begin{array}{c}
\alpha \\
\beta
\end{array}\right) x_{n}=0 .
$$

(2.1) has a nontrivial solution only if

$$
\lambda=\frac{\lambda_{n}\left(g_{2} \mu-\lambda_{n}\right)}{\left(f_{1} g_{2}-f_{2} g_{1}\right) \mu-\lambda_{n} f_{1}} .
$$

To see that $\Sigma_{A}=\{(\lambda, \mu)$ : (1.7) has a nontrivial solution at $(\lambda, \mu)\} \subseteq\{(\lambda, \mu)$ : $(\lambda, \mu)$ solves $(2.2)$ for some $\left.n \in \mathbf{Z}^{+}\right\}$, we proceed as follows. Observe that if $u$ and $v$ are nontrivial solutions to $(1.7)$, then

$$
\left(L-\lambda f_{1}\right)\left(L-\mu g_{2}\right) x=\mu \lambda f_{2} g_{1} x
$$


for $x=u, v$. It follows from (2.3) that $u$ and $v$ satisfy

$$
(L-c-d)(L-c+d) x=0,
$$

where

$$
c=c(\lambda, \mu)=\frac{\lambda f_{1}+\mu g_{2}}{2} \quad \text { and } \quad d=d(\lambda, \mu)=\sqrt{\left(\frac{\lambda f_{1}-\mu g_{2}}{2}\right)^{2}+\lambda \mu f_{2} g_{1}} .
$$

Hence either $c+d$ or $c-d$ is an eigenvalue of $L$. Since the eigenvalues of $L$ are all assumed to be real, it must be the case that $d^{2}(\lambda, \mu) \geq 0$ if $(\lambda, \mu) \in \Sigma_{A}$. Since the quadratic form $d^{2}(\lambda, \mu)$ is positive definite if $f_{2}^{2} g_{1}^{2}<f_{1} f_{2} g_{1} g_{2}$, we make the following additional assumption on $f$ and $g$ :

$$
f_{i}>0, \quad g_{i}>0 \quad \text { for } i=1,2 .
$$

With assumption (2.5), positive definiteness is equivalent to

$$
f_{2} g_{1}<f_{1} g_{2} \text {. }
$$

(2.5)-(2.6) will be assumed in the sequel. Then $c \pm d=\lambda_{n}$ yields (2.2). We have now established the following result.

Proposition 2.1. Let $\Sigma_{A}=\left\{(\lambda, \mu) \in \mathbf{R}^{2}:\right.$ (1.7) has a nontrivial solution at $(\lambda, \mu)\}$. Then

$$
\Sigma_{A}=\left\{(\lambda, \mu): \lambda=\frac{\lambda_{n}\left(g_{2} \mu-\lambda_{n}\right)}{\left(f_{1} g_{2}-f_{2} g_{1}\right) \mu-\lambda_{n} f_{1}}, n=1,2,3, \ldots\right\} .
$$

We now denote $\lambda^{(n)}$ by

$$
\lambda^{(n)}(\mu)=\frac{\lambda_{n}\left(g_{2} \mu-\lambda_{n}\right)}{\left(f_{1} g_{2}-f_{2} g_{1}\right) \mu-f_{1} \lambda_{n}} .
$$

Since $\lambda_{n} / g_{2}<f_{1} \lambda_{n} /\left(f_{1} g_{2}-f_{2} g_{1}\right)$ by $(2.5)-(2.6), \lambda^{(n)}$ is a linear fractional transformation. One readily observes the following simple facts:

(i) $\lambda^{(n)}(\mu)=0 \Leftrightarrow \mu=\lambda_{n} / g_{2}$.

(ii) $\lambda^{(n)}$ has a vertical asymptote at $\mu=f_{1} \lambda_{n} /\left(f_{1} g_{2}-f_{2} g_{1}\right)$.

$$
\lim _{\mu \rightarrow \pm \infty} \lambda^{(n)}(\mu)=\frac{\lambda_{n} g_{2}}{f_{1} g_{2}-f_{2} g_{1}} \quad(>0) .
$$

$$
\lambda^{(n)^{\prime}}(\mu)=\frac{-\lambda_{n}^{2} f_{2} g_{1}}{\left[\left(f_{1} g_{2}-f_{2} g_{1}\right) \mu-f_{1} \lambda_{n}\right]^{2}} .
$$

In order to analyze the bifurcation phenomena of (1.6), it is necessary that we examine in detail the intersections of the curves $\left\{\lambda^{(n)}: n=1,2, \ldots\right\}$.

A simple computation shows that if $\mu$ is such that $\lambda^{(n)}(\mu)=\lambda^{(m)}(\mu)$, where $n<m$, then

$$
\mu=\frac{\left(\lambda_{m}+\lambda_{n}\right) \pm \sqrt{\left(\lambda_{n}+\lambda_{m}\right)^{2}-4 f_{1} g_{2} \lambda_{n} \lambda_{m} /\left(f_{1} g_{1}-f_{2} g_{1}\right)}}{2 g_{2}} .
$$

Thus the curves $\lambda^{(n)}$ and $\lambda^{(m)}$ intersect if and only if

$$
\left(\frac{\lambda_{n}}{\lambda_{m}}\right)^{2}+\left[2-\frac{4 f_{1} g_{2}}{f_{1} g_{2}-f_{2} g_{1}}\right]\left(\frac{\lambda_{n}}{\lambda_{m}}\right)+1 \geq 0,
$$


which is equivalent to

$$
\frac{\lambda_{n}}{\lambda_{m}} \leq \frac{\sqrt{f_{1} g_{2}}-\sqrt{f_{2} g_{1}}}{\sqrt{f_{1} g_{2}}+\sqrt{f_{2} g_{1}}} .
$$

Observe now that if (2.7) holds, then

$$
\begin{aligned}
\mu_{-}^{*} & =\frac{\left(\lambda_{m}+\lambda_{n}\right)-\sqrt{\lambda_{m}^{2}+\left(2-4 f_{1} g_{2} /\left(f_{1} g_{2}-f_{2} g_{1}\right)\right) \lambda_{m} \lambda_{n}+\lambda_{n}^{2}}}{2 g_{2}} \\
& =\frac{\left(\lambda_{m}+\lambda_{n}\right)-\sqrt{\lambda_{m}^{2}-2\left(\left(f_{1} g_{2}+f_{2} g_{1}\right) /\left(f_{1} g_{2}-f_{2} g_{1}\right)\right) \lambda_{m} \lambda_{n}+\lambda_{n}^{2}}}{2 g_{2}} \\
& >\frac{\left(\lambda_{m}+\lambda_{n}\right)-\left[\lambda_{m}-\left(\left(f_{1} g_{2}+f_{2} g_{1}\right) /\left(f_{1} g_{2}-f_{2} g_{1}\right)\right) \lambda_{n}\right]}{2 g_{2}} \\
& =\frac{\lambda_{n} f_{1}}{f_{1} g_{2}-f_{2} g_{1}} .
\end{aligned}
$$

Similarly, if

$$
\mu_{+}^{*}=\frac{\left(\lambda_{m}+\lambda_{n}\right)+\sqrt{\lambda_{m}^{2}+\left(2-4 f_{1} g_{2} /\left(f_{1} g_{2}-f_{2} g_{1}\right)\right) \lambda_{n} \lambda_{m}+\lambda_{n}^{2}}}{2 g_{2}},
$$

then $\mu_{+}^{*}<\lambda_{m} f_{1} /\left(f_{1} g_{2}-f_{2} g_{1}\right)$. We may summarize as follows.

PROPOSITION 2.2. Let $n, m \in Z^{+}$with $n<m$. The curves $\lambda^{(n)}$ and $\lambda^{(m)}$ intersect if and only if (2.7) holds. Furthermore, if there is $\mu$ such that $\lambda^{n}(\mu)=$ $\lambda^{m}(\mu)$, then

$$
\frac{\lambda_{n} f_{1}}{f_{1} g_{2}-f_{2} g_{1}}<\mu<\frac{\lambda_{m} f_{1}}{f_{1} g_{2}-f_{2} g_{1}} .
$$

In particular, the following obtain:

(i) There are no $\mu \in \mathbf{R}$ and distinct $m, n, p \in Z^{+}$such that $\lambda^{m}(\mu)=\lambda^{n}(\mu)=$ $\lambda^{(p)}(\mu)$.

(ii) There are no $m \in Z^{+}, m>1$, and $\mu<\lambda_{1} f_{1} /\left(f_{1} g_{2}-f_{2} g_{1}\right)$ such that $\lambda^{m}(\mu)=\lambda^{1}(\mu)$.

We next make the following observation.

Proposition 2.3. Suppose $(\lambda, \mu)=\left(\lambda^{(n)}(\mu), \mu\right) \in \Sigma_{A}$. Then if $\left(\begin{array}{l}\alpha \\ \beta\end{array}\right) x_{n}$ is a solution of (1.7), corresponding to $\left(\lambda^{(n)}(\mu), \mu\right)$, where $L x_{n}=\lambda_{n} x_{n}, x_{n} \neq 0,\left(\begin{array}{l}\alpha \\ \beta\end{array}\right)$ is as follows:

(i) $\alpha \beta<0$ provided $\mu<0$ or $\mu>\lambda_{n} / g_{2}$;

(ii) $\alpha=0$ provided $\mu=\lambda_{n} / g_{2}$;

(iii) $\beta=0$ provided $\mu=0$;

(iv) $\alpha \beta>0$ provided $0<\mu<\lambda_{n} / g_{2}$.

PROOF. If $\left(\begin{array}{l}\alpha \\ \beta\end{array}\right)$ is as in the hypothesis, then $\left(\begin{array}{l}\alpha \\ \beta\end{array}\right)$ can readily be shown to satisfy

$$
\alpha \lambda_{n}=\alpha \lambda^{(n)}(\mu) f_{1}+\beta \lambda^{(n)}(\mu) f_{2}, \quad \beta \lambda_{n}=\alpha \mu g_{1}+\beta \mu g_{2} .
$$

(i)-(iv) then follow easily. 
DEFinition 2.4. Let $(\lambda, \mu) \in \Sigma_{A}$. Then we define the multiplicity of $(\lambda, \mu)$, denoted by $\operatorname{mult}(\lambda, \mu)$, by

$$
\operatorname{mult}(\lambda, \mu)=\operatorname{dim} \bigcup_{r \geq 1} \operatorname{kernel}\left\{\left(I-\left[\begin{array}{ll}
\lambda f_{1} L^{-1} & \lambda f_{2} L^{-1} \\
\mu g_{1} L^{-1} & \mu g_{2} L^{-1}
\end{array}\right]\right)^{r}\right\} .
$$

In particular, we say $(\lambda, \mu)$ is simple if $\operatorname{mult}(\lambda, \mu)=1$.

THEOREM 2.5. Suppose that $(\lambda, \mu)=\left(\lambda^{(n)}(\mu), \mu\right)$, where $\lambda_{n}$ is a simple eigenvalue of $L$. If $\lambda^{(n)}(\mu) \neq \lambda^{(m)}(\mu)$ for any $m \neq n$, then $(\lambda, \mu)$ is simple.

PROOF. If $\lambda=0$ or $\mu=0$, the result follows from well-known results for a single equation. Suppose then that $\lambda \neq 0, \mu \neq 0$ and that $c(\lambda, \mu)+d(\lambda, \mu)=\lambda_{n}$, while $c(\lambda, \mu)-d(\lambda, \mu)$ is not an eigenvalue of $L$, where $c$ and $d$ are as in (2.4). (The proof in case $c(\lambda, \mu)-d(\lambda, \mu)=\lambda_{n}$ is analogous to that which follows, and will be omitted.)

By the proof of Proposition 2.3,

$$
\text { kernel }\left(I-\left[\begin{array}{ll}
\lambda f_{1} L^{-1} & \lambda f_{2} L^{-1} \\
\mu g_{1} L^{-1} & \mu g_{2} L^{-1}
\end{array}\right]\right)
$$

is

$$
\left\langle\begin{array}{c}
x_{n} \\
\beta x_{n}
\end{array}\right\rangle
$$

where $\beta=\left(\lambda_{n}-\lambda f_{1}\right) / \lambda f_{2}$ and $L x_{n}=\lambda_{n} x_{n}$. It suffices then to assume

$$
L u-\lambda f_{1} u-\lambda f_{2} v=c x_{n}, \quad L v-\mu g_{1} u-\mu g_{2} v=c \beta x_{n}
$$

and show that $c=0$. Then

$$
\begin{aligned}
\left(L-\mu g_{2}\right)\left(L-\lambda f_{1}\right) u & =\lambda f_{2}\left(L-\mu g_{2}\right) v+c\left(L-\mu g_{2}\right) x_{n} \\
& =\lambda f_{2}\left[\mu g_{1} u+c \beta x_{n}\right]+c\left(\lambda_{n}-\mu g_{2}\right) x_{n} \\
& =\lambda \mu f_{2} g_{1} u+c\left(2 \lambda_{n}-\left(\lambda f_{1}+\mu g_{2}\right)\right) x_{n} .
\end{aligned}
$$

If $2 \lambda_{n}=\lambda f_{1}+\mu g_{2}$, then $c(\lambda, \mu)=\lambda_{n}$. Hence

$$
d^{2}(\lambda, \mu)=\left(\frac{\lambda f_{1}-\mu g_{2}}{2}\right)^{2}+\lambda \mu f_{2} g_{1}=0 .
$$

If such is the case, $\lambda \mu<0$. Hence

$$
\frac{\lambda^{2} f_{1}^{2}}{4}-\frac{\lambda \mu f_{1} g_{2}}{2}+\frac{\mu^{2} g_{2}^{2}}{4}=-\lambda \mu f_{2} g_{1}
$$

which implies

$$
\left(\frac{\lambda f_{1}+\mu g_{2}}{2}\right)^{2}=\lambda \mu\left(f_{1} g_{2}-f_{2} g_{1}\right)<0
$$

by (2.6), a contradiction. Thus $2 \lambda_{n}-\left(\lambda f_{1}+\mu g_{2}\right) \neq 0$.

So we have

$$
\left(L-\mu g_{2}\right)\left(L-\lambda f_{1}\right) u=\lambda \mu f_{2} g_{1} u+c\left(2 \lambda_{n}-\left(\lambda f_{1}+\mu g_{2}\right)\right) x_{n} .
$$

It follows that

$$
(L-c(\lambda, \mu)+d(\lambda, \mu))\left(L-\lambda_{n}\right) u=c\left(2 \lambda_{n}-\left(\lambda f_{1}+\mu g_{2}\right)\right) x_{n} .
$$


Since $L-c(\lambda, \mu)+d(\lambda, \mu)$ is invertible, it must be the case that

$$
\left(L-\lambda_{n}\right) u=\frac{c\left(2 \lambda_{n}-\left(\lambda f_{1}+\mu g_{2}\right)\right)}{\lambda_{n}-c(\lambda, \mu)+d(\lambda, \mu)} x_{n} .
$$

Since $\lambda_{n}$ is a simple eigenvalue of $L, c=0$, and the result is established.

3. Local bifurcation analysis. As previously noted, (1.6) is equivalent to an equation of the form

$$
e=A(\vec{\lambda}) e+H(\vec{\lambda}, e)
$$

where $(\vec{\lambda}, e) \in \mathbf{R}^{2} \times E, E$ a real Banach space, and $A$ and $H$ are as described in $\S 1$. If $\operatorname{dim} \Omega=1, \bar{\Omega}=[a, b]$, and $E$ may be taken as $\left(C_{0}^{1}[a, b]\right)^{2}=C_{0}^{1}[a, b] \times$ $C_{0}^{1}[a, b]$, where $C_{0}^{1}[a, b]$ is the space of continuously differentiable functions $u$ on $[a, b]$ such that $u(a)=0=u(b)$ with the usual norm (i.e. $\|u\|=\max _{x \in[a, b]}|u(x)|+$ $\left.\max _{x \in[a, b]}\left|u^{\prime}(x)\right|\right)$. If $\operatorname{dim} \Omega>1$, then $E$ will denote the cross product $\left[C_{0}^{1, \gamma}(\bar{\Omega})\right]^{2}$ of the space of continuously differentiable functions $u$ with Hölder continuous (of exponent $\gamma, 0<\gamma<1$ ) first partial derivatives such that $u \mid \partial \Omega \equiv 0$. (See $[\mathbf{1 7}$ or 23] for a precise definition of these spaces.)

In either case, a point $\left(\vec{\lambda}_{0}, \overrightarrow{0}\right)=\left(\lambda_{0}, \mu_{0}, 0,0\right)$ will be said to be a bifurcation point for (3.1) (or, equivalently (1.6)) provided that for any $\varepsilon>0$, there is $(\lambda, \mu, u, v) \in$ $\mathbf{R}^{2} \times E$ solving $(3.1)$ such that $(u, v) \neq(0,0)$ and $\left\|(\lambda, \mu, u, v)-\left(\lambda_{0}, \mu_{0}, 0,0\right)\right\|_{\mathbf{R}^{2} \times E}<$ $\varepsilon$. $B$ will denote the set $\left\{\vec{\lambda} \in \mathbf{R}^{2}:(\vec{\lambda}, 0)\right.$ is a bifurcation point for (3.1) $\}$.

Our first result is an immediate consequence of the fact that $H$ (see (3.1)) is higher-order in $e$.

Proposition 3.1. $B \subseteq \Sigma_{A}$.

We now aim to identify which members of $\Sigma_{A}$ are also contained in $B$. Our theorem on this topic is given considerable additional precision by Proposition 2.3.

THEOREM 3.2. (i) Let $(\lambda, \mu)=\left(\lambda^{(n)}(\mu), \mu\right) \in \Sigma_{A}$ with $\operatorname{mult}\left(\lambda^{(n)}(\mu), \mu\right)=1$. Then $\left(\lambda^{(n)}(\mu), \mu\right) \in B$.

(ii) Suppose all the eigenvalues of $L$ are simple. Then $B=\Sigma_{A}$.

(iii) Suppose $(\lambda, \mu)=\left(\lambda^{1}(\mu), \mu\right)$. Let $D=\left\{u \in C_{0}^{1, \gamma}(\bar{\Omega})\right.$ (respectively $\left.C_{0}^{1}[a, b]\right): u$ $>0$ on $\Omega$ and the outward normal $\partial u / \partial v<0$ on $\partial \Omega\}$. Then:

(a) If $\mu \in\left(0, \lambda_{1} / g_{2}\right)$, there is $\varepsilon>0$ such that if $(\tilde{\lambda}, \tilde{\mu}, u, v)$ is a solution of (3.1), $(u, v) \neq(0,0)$ and $\left\|(\tilde{\lambda}, \tilde{\mu}, u, v)-\left(\lambda^{(1)}(\mu), \mu, 0,0\right)\right\|_{\mathbf{R}^{2} \times E} \leq \varepsilon$, then $(u, v) \in$ $[D \times D] \cup[(-D) \times(-D)]$.

(b) If $\mu \in(-\infty, 0) \cup\left(\lambda_{1} / g_{2}, \infty\right)$, there is $\varepsilon>0$ such that if $(\tilde{\lambda}, \tilde{\mu}, u, v)$ is a solution of (3.1), $(u, v) \neq(0,0)$ and $\left\|(\tilde{\lambda}, \tilde{\mu}, u, v)-\left(\lambda^{(1)}(\mu), \mu, 0,0\right)\right\|_{\mathbf{R}^{2} \times E} \leq \varepsilon$, then $(u, v) \in[D \times(-D)] \cup[(-D) \times D]$.

(iv) Let $\bar{\Omega}=[a, b]$ and $(\lambda, \mu)=\left(\lambda^{(n)}(\mu), \mu\right), n>1$, with $\operatorname{mult}\left(\lambda^{(n)}(\mu), \mu\right)=$ 1. Let $D_{n}=\left\{u \in C_{0}^{1}[a, b]: u\right.$ has $n-1$ simple zeros on $(a, b), u^{\prime}(a)>0$ and $\left.(-1)^{n} u^{\prime}(b)>0\right\}$. Then:

(a) If $\mu \in\left(0, \lambda_{n} / g_{2}\right)$, there is $\varepsilon>0$ such that if $(\tilde{\lambda}, \tilde{\mu}, u, v)$ is a solution of (3.1), $(u, v) \neq(0,0)$ and $\left\|(\tilde{\lambda}, \tilde{\mu}, u, v)-\left(\lambda^{(n)}(\mu), \mu, 0,0\right)\right\|_{\mathbf{R}^{2} \times E} \leq \varepsilon$, then $(u, v) \in$ $\left[D_{n} \times D_{n}\right] \cup\left[\left(-D_{n}\right) \times\left(-D_{n}\right)\right]$. 
(b) If $\mu \in(-\infty, 0) \cup\left(\lambda_{n} / g_{2}, \infty\right)$, there is $\varepsilon>0$ such that if $(\tilde{\lambda}, \tilde{\mu}, u, v)$ is a solution of $(3.1),(u, v) \neq(0,0)$ and $\left\|(\tilde{\lambda}, \tilde{\mu}, u, v)-\left(\lambda^{(n)}(\mu), \mu, 0,0\right)\right\|_{\mathbf{R}^{2} \times E} \leq \varepsilon$, then $(u, v) \in\left[D_{n} \times\left(-D_{n}\right)\right] \cup\left[\left(-D_{n}\right) \times D_{n}\right]$.

PROOF. (i) is an application of a multiparameter extension of the Krasnosel'skiiRabinowitz Theorem (cf. $[\mathbf{3}, \mathbf{7}]$ ).

(ii) is a consequence of (i), Theorem 2.5, Proposition 2.2, and the fact that $B$ is closed.

We shall prove (iii) only in case (a). The verifications for the remaining part of (iii) and for (iv) are similar, and are omitted. Suppose then that (iii)(a) is false. Then there is a sequence $\left\{\left(\lambda_{m}, \mu_{m}, u_{m}, v_{m}\right)\right\}_{m=1}^{\infty} \subseteq \mathbf{R}^{2} \times E$ such that $\left(\lambda_{m}, \mu_{m}, u_{m}, v_{m}\right)$ is a solution of (3.1) (or, equivalently $\left.(1.6)\right),\left(u_{m}, v_{m}\right) \neq(0,0)$, $\left(u_{m}, v_{m}\right) \notin[D \times D] \cup[(-D) \times(-D)]$ and $\left(\lambda_{m}, \mu_{m}, u_{m}, v_{m}\right) \rightarrow\left(\lambda^{1}(\mu), \mu, 0,0\right)$ as $m \rightarrow \infty$. The compactness of the operators $A(\lambda)$ and $H(\lambda, \cdot)$ imply the existence of a subsequence $\left\{\left(\lambda_{m_{i}}, \mu_{m_{i}}, u_{m_{i}}, v_{m_{i}}\right)\right\}$ such that

$$
\left(\lambda_{m_{i}}, \mu_{m_{i}}, \frac{u_{m_{i}}}{\left\|\left(u_{m_{i}}, v_{m_{i}}\right)\right\|_{E}}, \frac{v_{m_{i}}}{\left\|\left(u_{m_{i}}, v_{m_{i}}\right)\right\|_{E}}\right) \rightarrow\left(\lambda^{(1)}(\mu), \mu, \bar{u}, \bar{v}\right)
$$

where $\left(\lambda^{(1)}(\mu), \mu, \bar{u}, \bar{v}\right)$ solves $(1.7)$ and $(\bar{u}, \bar{v}) \neq(0,0)$. Proposition 2.3 implies $(\bar{u}, \bar{v}) \in[D \times D] \cup[(-D) \times(-D)]$. Without loss of generality, assume $(\bar{u}, \bar{v}) \in D \times D$. Since $D \times D$ is open in $E$,

$$
\left(\frac{u_{m_{i}}}{\left\|\left(u_{m_{i}}, v_{m_{i}}\right)\right\|_{E}}, \frac{v_{m_{i}}}{\left\|\left(u_{m_{i}}, v_{m_{i}}\right)\right\|_{E}}\right) \in D \times D
$$

for $i$ sufficiently large. But $(a, b) \in D \times D$ implies $t(a, b) \in D \times D$ for $t>0$, a contradiction.

(iii) and (iv) of Theorem 3.2 have an immediate noteworthy consequence. We now state the appropriate result only in case (iii)(a). The remaining results are completely analogous, and their statements are left to the reader.

Let $S=\overline{\left\{(\lambda, \mu, u, v) \in \mathbf{R}^{2} \times E:(\lambda, \mu, u, v) \text { solves }(3.1) \text { and }(u, v) \neq(0,0)\right\}}$. By a continuum we shall mean a closed, connected set.

COROLlaRY 3.3. Let $C \subseteq S$ be a continuum such that $\left(\lambda^{1}(\mu), \mu, 0,0\right) \in C$, where $0<\mu<\lambda_{1} / g_{2}$. Suppose $C^{\prime} \subseteq C \backslash(B \times\{\overrightarrow{0}\})$ is a subcontinuum of $C$ such that $C^{\prime} \subseteq \overline{B\left(\lambda^{1}(\mu), \mu, 0,0 ; \varepsilon\right)}$, where $\varepsilon$ is as in Theorem 3.2(iii)(a). Then either

$$
C^{\prime} \subseteq \mathbf{R}^{2} \times D \times D \quad \text { or } \quad C^{\prime} \subseteq \mathbf{R}^{2} \times(-D) \times(-D) .
$$

Theorem 3.2 gives a qualitative description in certain instances of the nontrivial solutions in an $\mathbf{R}^{2} \times E$ neighborhood of a bifurcation point. We now use a LyapunovSchmidt type procedure by which we may, under appropriate conditions, obtain a count on the number of such solutions for particular parameter values (cf. [26]). For the remainder of this section, we assume that $A=L$ (cf. (1.6)), and, for the sake of convenience, employ much of the notation of [26]. In addition, if $\Omega \subseteq \mathbf{R}^{N}$ for $N>1$, we shall take $\left[C_{0}(\bar{\Omega}]^{2}\right.$ as our Banach space $E$ for the remainder of this section. Our purpose here is to facilitate use of the contraction mapping principle. Since, as is well known $[\mathbf{1 7}], L^{-1}$ extends to a compact operator from $C_{0}(\bar{\Omega}) \rightarrow C_{0}(\bar{\Omega})$, there is no difficulty in making this change. Furthermore, no essential information is lost in the process. Any solution to (3.1) which belongs to $\left[C_{0}(\bar{\Omega})\right]^{2}$ is necessarily a 
classical solution by the smoothness of the nonlinearity and the regularity theory for elliptic partial differential equations. The solution set is therefore the same in $\left[C_{0}(\bar{\Omega})\right]^{2}$ as in $\left[C_{0}^{1, \gamma}(\bar{\Omega})\right]^{2}$. Moreover, the topological properties of $\left[C_{0}^{1, \gamma}(\bar{\Omega})\right]^{2}$ used in Theorem 3.2 (namely, that the set $D$ is open in $C_{0}^{1, \gamma}(\bar{\Omega})$ ) are unnecessary for the following arguments.

Write (1.6) as

$$
L u=\lambda\left[f_{1} u+f_{2} v+\tilde{f}(u, v)\right], \quad L v=\mu\left[g_{1} u+g_{2} v+\tilde{g}(u, v)\right],
$$

where $\tilde{f}, \tilde{g}$ are higher-order and $u|\partial \Omega \equiv 0 \equiv v| \partial \Omega$. Letting $G=L^{-1},(3.2)$ can be rewritten

$$
\phi=\Lambda A \mathcal{G} \phi+\Lambda \mathcal{G} N(\phi)
$$

where $\phi=\left(\begin{array}{l}u \\ v\end{array}\right), E=\left[C_{0}(\bar{\Omega})\right]^{2}$ (or $\left[C_{0}^{1}(\bar{\Omega})\right]^{2}$, as indicated above),

$$
\Lambda=\left(\begin{array}{cc}
\lambda & 0 \\
0 & \mu
\end{array}\right), \quad A=\left(\begin{array}{ll}
f_{1} & f_{2} \\
g_{1} & g_{2}
\end{array}\right), \quad \mathcal{G}=\left(\begin{array}{cc}
G & 0 \\
0 & G
\end{array}\right), \quad N(\phi)=\left(\begin{array}{l}
\tilde{f}(u, v) \\
\tilde{g}(u, v)
\end{array}\right) .
$$

Let $\Lambda^{*}=\left(\begin{array}{cc}\lambda^{*} & 0 \\ 0 & \mu^{*}\end{array}\right)$ be such that $\operatorname{mult}\left(\lambda^{*}, \mu^{*}\right)=1$ and $\lambda^{*} \neq 0, \mu^{*} \neq 0$. Let $N\left(I-\Lambda^{*} A \mathcal{G}\right)=\left[\phi^{*}\right]$, with $\left\|\phi^{*}\right\|_{E}=1$. Let

$$
\tau=\Lambda-\Lambda^{*}=\left(\begin{array}{cc}
\lambda-\lambda^{*} & 0 \\
0 & \mu-\mu^{*}
\end{array}\right)=\left(\begin{array}{cc}
\tau_{1} & 0 \\
0 & \tau_{2}
\end{array}\right)
$$

Then (3.3) is equivalent to

$$
\phi-\Lambda^{*} A \phi=\tau A \phi+\Lambda \mathcal{G N}(\phi),
$$

where $A=A \mathcal{G}$. Since $A$ is compact on $E$ and mult $\Lambda^{*}=1, E=N \oplus R$, where $N$ and $R$ are nullspace and range of $I-\Lambda^{*} A$, respectively. Thus we may define a linear homeomorphism $T: E \rightarrow E$ by

$$
T \phi=\left(I-\Lambda^{*} \AA\right) \phi+\langle\phi, \gamma\rangle \phi^{*}
$$

where $\gamma \in E^{*}$ (the dual space of $E$ ) is such that $\left\langle\phi^{*}, \gamma\right\rangle=\gamma\left(\phi^{*}\right)=1$ and $\langle x, \gamma\rangle=0$ for all $x \in R$. (For example, if $L$ is selfadjoint when viewed as an operator on $L^{2}(\Omega), f_{2}=g_{1}$ and

$$
\int_{\Omega}\left(\frac{\left(u^{*}\right)^{2}}{\lambda^{*}}+\frac{\left(v^{*}\right)^{2}}{\mu^{*}}\right) d x \neq 0
$$

where $\phi^{*}=\left(\begin{array}{l}u^{*} \\ v^{*}\end{array}\right)$, then $\gamma$ may be realized as

$$
\gamma\left(\begin{array}{l}
u \\
v
\end{array}\right)=\frac{1}{k} \int_{\Omega}\left(u \frac{u^{*}}{\lambda^{*}}+v \frac{v^{*}}{\mu^{*}}\right) d x
$$

with $k=\int_{\Omega}\left(\left(u^{*}\right)^{2} / \lambda^{*}+\left(v^{*}\right)^{2} / \mu^{*}\right) d x$. $)$

(3.4) is thus equivalent to

$$
\begin{gathered}
\phi=\alpha \phi^{*}+T^{-1}[\tau \not \mathcal{A} \phi+\Lambda \mathcal{G N}(\phi)], \\
\alpha=\langle\phi, \gamma\rangle .
\end{gathered}
$$

As in $[\mathbf{2 6}]$, we have the following result. 
LEMMA 3.4. There are positive $\omega, \sigma, \delta$ such that if $|\alpha|<\omega$ and $\left\|\left(\tau_{1}, \tau_{2}\right)\right\|_{\mathbf{R}^{2}}<\sigma$, the right-hand side of (3.6) is a contraction mapping of $\overline{B(0, \delta)} \subseteq E$ into $\overline{B(0, \delta)}$.

Let $\hat{\phi}\left(\alpha, \tau_{1}, \tau_{2}\right)$ denote the unique fixed point for (3.6). In particular, $\hat{\phi}\left(0, \tau_{1}, \tau_{2}\right)$ $=0$ for all $\tau_{1}, \tau_{2}$ with $\left\|\left(\tau_{1}, \tau_{2}\right)\right\|_{\mathbf{R}^{2}}<\sigma$, where $\sigma$ is as in Lemma 3.4.

Solvability of (3.6) $-(3.7)$ can readily be shown equivalent to

$$
\left\langle T^{-1}[\tau \mathcal{A} \hat{\phi}+\Lambda \mathcal{G N}(\hat{\phi})], \gamma\right\rangle=0
$$

A simple computation will show that for all $x \in E,\left\langle T^{-1} x, \gamma\right\rangle=\langle x, \gamma\rangle$ and $\left\langle\Lambda^{*} A x, \gamma\right\rangle=\langle x, \gamma\rangle$. Thus (3.8) may be simplified to show solvability of (1.6) is equivalent to

$$
\left\langle A^{-1} \Lambda^{*-1}(\tau A \hat{\phi}+\Lambda N(\hat{\phi})), \gamma\right\rangle=0
$$

Now let

$$
S\left(\alpha, \tau_{1}, \tau_{2}\right)=\left\langle A^{-1} \Lambda^{*-1}(\tau A \hat{\phi}+\Lambda N(\hat{\phi})), \gamma\right\rangle .
$$

Then $S$ is smooth in $\left(\alpha, \tau_{1}, \tau_{2}\right)$. If $\left(\partial^{i} S / \partial \alpha^{i}\right)(0,0,0)=0$ for $i=0,1, \ldots, r-1$ and $\left(\partial^{r} S / \partial \alpha^{r}\right)(0,0,0) \neq 0$, the Malgrange Preparation Theorem [18] may be utilized to show

$$
S\left(\alpha, \tau_{1}, \tau_{2}\right)=\left[\alpha^{r}+\rho_{1}\left(\tau_{1}, \tau_{2}\right) \alpha^{r-1}+\cdots+\rho_{r-1}\left(\tau_{1}, \tau_{2}\right) \alpha+\rho_{r}\left(\tau_{1}, \tau_{2}\right)\right] E\left(\alpha, \tau_{1}, \tau_{2}\right),
$$

where $\rho_{i}\left(\tau_{1}, \tau_{2}\right)$ is smooth in $\tau_{1}$ and $\tau_{2},\left|\tau_{1}\right|,\left|\tau_{2}\right|$ sufficiently small with $\rho_{i}(0,0)=$ $0, i=1,2, \ldots, r$, and $E\left(\alpha, \tau_{1}, \tau_{2}\right)$ is smooth in $\alpha, \tau_{1}$ and $\tau_{2},|\alpha|,\left|\tau_{1}\right|,\left|\tau_{2}\right|$ small with $E(0,0,0) \neq 0$. Furthermore, one may readily observe that $\rho_{r}\left(\tau_{1}, \tau_{2}\right) \equiv 0$. Hence we have the following result.

THEOREM 3.5. If, in $(3.10),\left(\partial^{i} S / \partial \alpha^{i}\right)(0,0,0)=0, i=0,1, \ldots, r-1$ and $\left(\partial^{r} S / \partial \alpha^{r}\right)(0,0,0) \neq 0$, then there are positive $\omega^{\prime}<\omega$ and $\sigma^{\prime}<\sigma$, with $\omega, \sigma, \delta$ as in Lemma 3.4, such that (3.11) holds. Thus the number of solutions $(\lambda, \mu, e)$ of (1.6) with $0<\|e\|<\delta$ and $\left\|(\lambda, \mu)-\left(\lambda^{*}, \mu^{*}\right)\right\|_{\mathbf{R}^{2}}<\sigma^{\prime}$ is the number of distinct real nonzero roots of the polynomial

$$
\alpha^{r-1}+\rho_{1}\left(\tau_{1}, \tau_{2}\right) \alpha^{r-2}+\cdots+\rho_{r-2}\left(\tau_{1}, \tau_{2}\right) \alpha+\rho_{r-1}\left(\tau_{1}, \tau_{2}\right),
$$

where $\tau_{1}=\lambda-\lambda^{*}$ and $\tau_{2}=\mu-\mu^{*}$, with $|\alpha|<\omega^{\prime}$.

Note that this number $r$ may be determined from (3.9) with $\tau_{1}=0=\tau_{2}$ :

$$
\left\langle A^{-1} N(\hat{\phi}(\alpha, 0,0)), \gamma\right\rangle=0 \text {. }
$$

Suppose now that $f$ and $g$ are analytic and that for $w, z \in \mathbf{R}$ with $|w|,|z|$ sufficiently small, $\tilde{f}(w, z)=\sum_{l+m \geq 2} f_{l m} w^{l} z^{m}$ and $\tilde{g}(w, z)=\sum_{l+m \geq 2} g_{l m} w^{l} z^{m}$. Then it is the case that

$$
r \geq s=\min \left\{l+m: l+m \geq 2 \text { and } f_{l m} \neq 0 \text { or } g_{l m} \neq 0\right\} .
$$

We now give conditions under which $r=s$. Observe that $\hat{\phi}(\alpha, 0,0)=$ $\sum_{i \geq 1} \phi_{i 00} \alpha^{i}$ with $\phi_{100}=\phi^{*}=\left(\begin{array}{l}u^{*} \\ v^{*}\end{array}\right)$ and that in the expansion of the left-hand 
side of (3.12) terms involving $\alpha^{s}$ occur only in $\left\langle A^{-1} N\left(\alpha \phi^{*}\right), \gamma\right\rangle$. One may readily show that

$$
\left\langle A^{-1} N\left(\alpha \phi^{*}\right), \gamma\right\rangle=\frac{1}{f_{1} g_{2}-f_{2} g_{1}}\left\langle\sum_{l+m \geq 2}\left(g_{2} f_{l m}-f_{2} g_{l m}\right) u^{* l} v^{* m} \alpha^{l+m}\left(f_{1} g_{l m}-g_{1} f_{l m}\right) u^{* l} v^{* m} \alpha^{l+m}, \gamma\right\rangle .
$$

From (3.13), we see that $r=s$ provided

$$
\left.\left\langle\sum_{l+m=s}\left(g_{2} f_{l m}-f_{2} g_{l m}\right) u^{* l} v^{* m} g_{l m}-g_{1} f_{l m}\right) u^{* l} v^{* m}, \gamma\right\rangle \neq 0 .
$$

In case $A$ is symmetric, $L$ is selfadjoint (with respect to $L^{2}(\Omega)$ ) and

$$
\int_{\Omega}\left(\frac{\left(u^{*}\right)^{2}}{\lambda^{*}}+\frac{\left(v^{*}\right)^{2}}{\mu^{*}}\right) d x \neq 0
$$

(3.14) reduces to

$\left(\int_{\Omega}\left(u^{*}\right)^{s+1}\right) \cdot \sum_{l+m=s} \varepsilon\left(\mu^{*}\right)^{m}\left[\left(g_{2} f_{l m}-f_{2} g_{l m}\right)+\left(\frac{\lambda^{*}}{\mu^{*}}\right) \varepsilon\left(\mu^{*}\right)\left(f_{1} g_{l m}-f_{2} f_{l m}\right)\right] \neq 0$,

where $v^{*}=\varepsilon\left(\mu^{*}\right) u^{*}$.

4. Multidimensionality questions. In problems in which more than one parameter appears, such as (1.6), a new aspect of study arises quite naturally in addition to the concerns of $\S 3$. Namely, there is an obvious expectation that the set of nontrivial solutions to the problem forms a higher dimensional set of some sort. As a simple example (which also illustrates the third alternative to Theorem 2.7 in $[8])$, consider the equation

$$
x=\left(\left|\lambda_{1}\right|+\left|\lambda_{2}\right|\right) x+\left(1+\lambda_{1}^{2}+\lambda_{2}^{2}\right) x^{3},
$$

with $\lambda_{1} \in \mathbf{R}_{1}, \lambda_{2} \in \mathbf{R}$ viewed as parameters, and $x \in \mathbf{R}$. Then (4.1) is of the form (1.4)-(1.5) and has solutions $\left(\lambda_{1}, \lambda_{2}, 0\right), \lambda_{1} \in \mathbf{R}, \lambda_{2} \in \mathbf{R}$, and $\left(\lambda_{1}, \lambda_{2}, x\right)$ satisfying

$$
x^{2}=\frac{1-\left(\left|\lambda_{1}\right|+\left|\lambda_{2}\right|\right)}{1+\lambda_{1}^{2}+\lambda_{2}^{2}} .
$$

The principal results on this multidimensionality of bifurcation surfaces to date are the global results of Alexander and Antman $[\mathbf{3}, \mathbf{4}]$ and Fitzpatrick, Massabo and Pejsachowicz $[\mathbf{1 4}, \mathbf{1 5}, \mathbf{1 6}]$. The main theorem of $[\mathbf{3}]$ is applicable in the context of (1.6) (as (1.5) holds for (1.6) with $k=1-\mathrm{cf}$. [7]), and, as such may be expressed:

THEOREM 4.1. Consider (1.6), or equivalently (3.1). Let $\left(\lambda_{0}, \mu_{0}\right) \in \mathbf{R}^{2}$ be a simple generalized characteristic value of (1.7). Then there emanates from $\left(\lambda_{0}, \mu_{0}, 0,0\right) \in \mathbf{R}^{2} \times E$ a subset $S_{\left(\lambda_{0}, \mu_{0}\right)} \subseteq S$ which is global in the sense of Theorem 2.2 of $[\mathbf{3}]$ and which has the following properties:

(i) $S_{\left(\lambda_{0}, \mu_{0}\right)} \cap[S \backslash(B \times\{0\})]$ is connected. 
(ii) $S_{\left(\lambda_{0}, \mu_{0}\right)} \cap[S \backslash(B \times\{0\})]$ has topological dimension $\geq 2$ at each point. (Note: For a precise definition of topological dimension, see Alexander and Antman [3] and the references therein.)

REMARK 4.2. Theorem 4.1 does not assert that if $\hat{S}_{\left(\lambda_{0}, \mu_{0}\right)}$ is the component of $S$ containing $\left(\lambda_{0}, \mu_{0}, 0,0\right)$, then $\hat{S}_{\left(\lambda_{0}, \mu_{0}\right)} \backslash(B \times\{0\})$ has topological dimension $\geq 2$ at every point. It is the case, however, that along one-dimensional restrictions of parameter space, $S_{\left(\lambda_{0}, \mu_{0}\right)}$ is described by the global Rabinowitz bifurcation theory $[3,7]$. As this fact will be of significance in the subsequent sections of this paper, we make this observation precise.

DEFINITION 4.3. A continuous map $h: \mathbf{R} \rightarrow \mathbf{R}^{2}$ is a proper crossing of changing degree at $\left(\lambda_{0}, \mu_{0}\right) \in \mathbf{R}^{2}$ relative to (1.6) (or (3.1)) if the following conditions hold:

(i) $h(0)=\left(\lambda_{0}, \mu_{0}\right)$ and $|h(t)| \rightarrow \infty$ as $t \rightarrow \pm \infty$.

(ii) If $\gamma>0$, there is a neighborhood $V$ of $\left(\lambda_{0}, \mu_{0}\right)$ in $\mathbf{R}^{2}$ such that

$$
h^{-1}(V \cap h(\mathbf{R})) \subseteq(-\gamma, \gamma)
$$

(iii) There is a number $\varepsilon_{h}>0$ such that:

(a) The Leray-Schauder degree $\operatorname{deg}_{\mathrm{LS}}(I-A(h(t)), B(0,1), 0)$ is defined for all $t$ such that $|t|<\varepsilon_{h}$ and $t \neq 0$, where $A(\lambda, \mu)$ is given by $(1.8)$;

(b)

$$
\operatorname{deg}_{\mathrm{LS}}(I-A(h(\tau)), B(0,1), 0)=\operatorname{sgn}(\tau \beta) \cdot \operatorname{deg}_{\mathrm{LS}}(I-A(h(\beta)), B(0,1), 0),
$$

where $\tau, \beta \in\left(-\varepsilon_{h}, \varepsilon_{h}\right), \tau \neq 0, \beta \neq 0$.

THEOREM 4.4. If $\left(\lambda_{0}, \mu_{0}\right) \in \mathbf{R}^{2}$ is as in Theorem 4.1 and $h$ is a smooth proper crossing of changing degree at $\left(\lambda_{0}, \mu_{0}\right)$, and if $S_{\left(\lambda_{0}, \mu_{0}\right)}^{h}=S_{\left(\lambda_{0}, \mu_{0}\right)} \cap[h(\mathbf{R}) \times E]$, then $S_{\left(\lambda_{0}, \mu_{0}\right)}^{h}$ satisfies one of the following conditions:

(i) $S_{\left(\lambda_{0}, \mu_{0}\right)}^{h}$ is unbounded.

(ii) $S_{\left(\lambda_{0}, \mu_{0}\right)}^{h}$ contains $\left(\lambda^{*}, \mu^{*}, 0,0\right),\left(\lambda^{*}, \mu^{*}\right) \neq\left(\lambda_{0}, \mu_{0}\right)$, with $\left(\lambda^{*}, \mu^{*}\right)$ a generalized characteristic value of (1.8).

REMARK 4.5. Cf. [3, p. 349]. See also $\S 2$ of [7].

While the methods used to establish the main result (Theorem 2.2) of $[\mathbf{3}]$ are not of primary interest here, two features are noteworthy. First, techniques from algebraic topology in addition to the topological degree of Leray and Schauder were employed. Second, no differentiablity assumptions with respect to $(u, v)$ were made, other than those on (1.4). Of course, such results may be sharpened when more precise information is given, as in (1.6).

Therefore let us now assume that $A=L$ in (1.6), that $f$ and $g$ are analytic and that (3.14) is valid. Consider the Malgrange polynomial

$$
M\left(\alpha, \tau_{1}, \tau_{2}\right)=\alpha^{s-1}+\rho_{1}\left(\tau_{1}, \tau_{2}\right) \alpha^{s-2}+\cdots+\rho_{s-1}\left(\tau_{1}, \tau_{2}\right)
$$

where $\tau_{1}=\lambda-\lambda^{*}, \tau_{2}=\mu-\mu^{*},\left(\lambda^{*}, \mu^{*}\right)$ a simple generalized characteristic value of (1.8), as in the preceding section. For our purposes, we restrict $\tau_{1}, \tau_{2}$ in $(4.3)$ to be real numbers such that $\left|\left(\tau_{1}, \tau_{2}\right)\right|<\sigma^{\prime}$, where $\sigma^{\prime}>0$ is as in the statement of Theorem 3.5. Let $r\left(\tau_{1}, \tau_{2}\right) \in \mathbf{R}-\{0\}$ be such that $\left|r\left(\tau_{1}, \tau_{2}\right)\right|<w^{\prime}, w^{\prime}>0$ as in Theorem 3.5, $M\left(r\left(\tau_{1}, \tau_{2}\right), \tau_{1}, \tau_{2}\right)=0$ but $M_{\alpha}\left(r\left(\tau_{1}, \tau_{2}\right), \tau_{1}, \tau_{2}\right) \neq 0$. Then if $S\left(\alpha, \tau_{1}, \tau_{2}\right)$ is as in $(3.10), S\left(r\left(\tau_{1}, \tau_{2}\right), \tau_{1}, \tau_{2}\right)=0$ but $S_{\alpha}\left(r\left(\tau_{1}, \tau_{2}\right), \tau_{1}, \tau_{2}\right) \in \mathbf{R}-\{0\}$. 
The Implicit Function Theorem then implies that $S \backslash\left(\mathbf{R}^{2} \times\{0\}\right)$ is a smooth twodimensional manifold in a neighborhood of $\left(\lambda, \mu, \hat{\phi}\left(r\left(\lambda-\lambda^{*}, \mu-\mu^{*}\right), \lambda-\lambda^{*}, \mu-\mu^{*}\right)\right)$. In particular, there are $\sigma^{\prime \prime}, 0<\sigma^{\prime \prime}<\sigma^{\prime}$, and a smooth function $\tilde{r}: B\left(\lambda, \mu ; \sigma^{\prime \prime}\right) \rightarrow$ $\mathbf{R}-\{0\}$ such that $\tilde{r}(\lambda, \mu)=r\left(\lambda-\lambda^{*}, \mu-\mu^{*}\right)$ and such that if $\left(\lambda^{\prime}, \mu^{\prime}\right) \in B\left(\lambda, \mu, \sigma^{\prime \prime}\right)$, then

$$
\left(\lambda^{\prime}, \mu^{\prime}, \hat{\phi}\left(\hat{r}\left(\lambda^{\prime}, \mu^{\prime}\right), \lambda^{\prime}-\lambda^{*}, \mu^{\prime}-\mu^{*}\right)\right) \in S-\left(\mathbf{R}^{2} \times\{0\}\right) .
$$

We have the following result.

THEOREM 4.6. Suppose $A=L$ in (1.6), $f$ and $g$ are analytic, and that $\left(\lambda^{*}, \mu^{*}\right)$ is a simple generalized characteristic value for (1.8). Suppose that (3.14) holds with $r=s$ and that $(\lambda, \mu) \in \mathbf{R}^{2}$ with $\left\|(\lambda, \mu)-\left(\lambda^{*}, \mu^{*}\right)\right\|<\sigma^{\prime}$, where $\sigma^{\prime}$ is as in Theorem 3.6. Then if $r_{i}\left(\lambda-\lambda^{*}, \mu-\mu^{*}\right), i=1,2, \ldots, m$, are simple real zeros of (4.3) such that $0<\left|r_{i}\left(\lambda-\lambda^{*}, \mu-\mu^{*}\right)\right|<\omega^{\prime}, i=1,2, \ldots, m$, and $\omega^{\prime}$ is as in Theorem $3.6, m \leq s-1$ and $\left[S \backslash\left(\mathbf{R}^{2} \times\{0\}\right)\right] \cap\left[B\left(\lambda, \mu ; \sigma^{\prime \prime}\right) \times B(0, \delta)\right]$ contains $m$ smooth two-dimensional manifolds $D_{i}, i=1, \ldots, m$, with $\left(\lambda, \mu, \hat{\phi}\left(r_{i}\left(\lambda-\lambda^{*}, \mu-\mu^{*}\right), \lambda-\lambda^{*}, \mu-\mu^{*}\right)\right) \in D_{i}$, where $\sigma^{\prime \prime} \in\left(0, \sigma^{\prime}\right)$ and $\delta$ is as in Theorem 3.5.

The number $m$ above can actually be seen to be 0,1 or 2 , provided $\sigma^{\prime}$ and $\omega^{\prime}$ are sufficiently small. That such is the case can be seen by combining Theorem 3.5 with the constructive bifurcation theorem of Rabinowitz (Theorem 1.19 of [23] or its generalization to several parameters, due to Alexander and Antman, Theorem 3.12 of $[3]$ ). As a consequence, the cases $r=s=2$ and $r=s=3$ are the most important, and merit separate consideration.

Suppose now (3.14) holds with $r=s=2$. In this case $W\left(\alpha, \tau_{1}, \tau_{2}\right)=\alpha+$ $\rho_{1}\left(\tau_{1}, \tau_{2}\right)$. Thus for $(\lambda, \mu) \in B\left(\lambda^{*}, \mu^{*} ; \gamma\right) \backslash \Sigma_{A}, \gamma$ is sufficiently small, (1.6) has at most one nontrivial solution $\phi \in E$ with $\|\phi\|<\delta$. Consider now the one-dimensional restriction of the parameters along normals to $\Sigma_{A}$ for $\left(\lambda^{\prime}, \mu^{\prime}\right) \in B\left(\lambda^{*}, \mu^{*} ; \gamma\right) \cap \Sigma_{A}$, where we assume $\gamma$ is sufficiently small so that $\operatorname{mult}\left(\lambda^{\prime}, \mu^{\prime}\right)=1$ for all $\left(\lambda^{\prime}, \mu^{\prime}\right) \in$ $B\left(\lambda^{*}, \mu^{*} ; \gamma\right) \cap \Sigma_{A}$. One may then adapt Lemma 1.24 and Theorem 1.25 of [24] of Theorem 2 of $[\mathbf{1 1}]$ to assert the existence of two subcontinua of nontrivial solutions (with parameter values along this one-dimensional restriction) meeting only at $\left(\lambda^{\prime}, \mu^{\prime} ; 0\right)$. The following result then obtains.

Corollary 4.7. Suppose (3.14) holds with $r=s=2$. Then if $\left(\lambda^{*}, \mu^{*}\right)$ is as Theorem 4.6 and $(\lambda, \mu) \in B\left(\lambda^{*}, \mu^{*} ; \gamma\right)-\Sigma_{A}, \gamma>0$ and sufficiently small, there is a unique $e=\hat{\phi}\left(-\rho_{1}\left(\lambda-\lambda^{*}, \mu-\mu^{*}\right), \lambda-\lambda^{*}, \mu-\mu^{*}\right)$ such that $0<\|e\|<\delta$ and $(\lambda, \mu, e) \in S$, where $\delta$ is as in Theorem 3.5 .

COROllary 4.8. Suppose (3.14) holds with $r=s=3$ and $\left(\lambda^{*}, \mu^{*}\right)$ is as in Theorem 4.6. Then one of the following obtains:

(i) If $\rho_{2}\left(\tau_{1}, \tau_{2}\right) \equiv 0$, then if $(\lambda, \mu) \in B\left(\lambda^{*}, \mu^{*} ; \gamma\right) \backslash \Sigma_{A}, \gamma>0$ and sufficiently small, there is a unique $e=\phi\left(-\rho_{1}\left(\lambda-\lambda^{*}, \mu-\mu^{*}\right), \lambda-\lambda^{*}, \mu-\mu^{*}\right)$ such that $0<\|e\|<\delta$ and $(\lambda, \mu, e) \in S$, where $\delta$ is as in Theorem 3.5. (ii) If $\rho_{2}\left(\tau_{1}, \tau_{2}\right) \not \equiv 0$, there is a component $V$ of $B\left(\lambda^{*}, \mu^{*} ; \gamma\right) \backslash \Sigma_{A}, \gamma>0$ and sufficiently small, such that $S \cap\left[\{(\lambda, \mu)\} \times B(0, \delta)^{0}\right] \neq \phi$ for all $(\lambda, \mu) \in V$, where $B(0 ; \delta)^{0}=B(0 ; \delta) \backslash\{0\}$. Furthermore, $S \cap\left(V \times B(0, \delta)^{0}\right)$ is a two-dimensional set.

If (ii) above holds and

$$
S \cap\left[\left(B\left(\lambda^{*}, \mu^{*}, \gamma\right)-\left(\Sigma_{A} \cup V\right)\right) \times B(0, \delta)^{0}\right]=\phi,
$$


then $(\lambda, \mu) \in V$ implies $\left.S \cap\{(\lambda, \mu)\} \times B(0, \delta)^{0}\right]=\left\{\left(\lambda, \mu, e_{1}\right),\left(\lambda, \mu, e_{2}\right)\right\}, e_{1} \neq e_{2}$; i.e. $S \cap\left(V \times B(0, \delta)^{0}\right)$ is the union of two nonintersecting two-dimensional manifolds.

PROOF. Here $W\left(\alpha, \tau_{1}, \tau_{2}\right)=\alpha^{2}+\rho_{1}\left(\tau_{1}, \tau_{2}\right) \alpha+\rho_{2}\left(\tau_{1}, \tau_{2}\right)$. (i) follows as in Corollary 4.7. If $\rho_{2}\left(\tau_{1}, \tau_{2}\right) \not \equiv 0$, then it cannot vanish on any open subset of $B\left(\lambda^{*}, \mu^{*} ; \gamma\right)$ by principles of analytic continuation [12]. Lemma 1.24 and Theorem 1.25 of [24] may then be used to complete the proof.

5. Determining global alternatives via the linearized system. Consider again (1.6) (or, equivalently, (3.1)). Theorem 4.4 asserts that if mult $\left(\lambda_{0}, \mu_{0}\right)=$ 1 and $h$ is any smooth unbounded curve in $\mathbf{R}^{2}$ which meets $\Sigma_{A}$ transversely at $\left(\lambda_{0}, \mu_{0}\right)$, then $S_{\left(\lambda_{0}, \mu_{0}\right)}^{h}$ conforms to the global Rabinowitz alternatives.

Recall that (3.1) is a special case of (1.4)-(1.5), as is example (4.1). Example (4.1) illustrates a situation where the second alternative of Theorem 4.4 always obtains. As perhaps should be expected, such is not the case with (3.1), as we now demonstrate. Let us suppose that all the eigenvalues of $L$ are simple. Then Theorem 2.5 asserts that if $\lambda^{(n)}$ is as in $\S 2$, mult $\left(\lambda^{(n)}(\mu), \mu\right)=1$ except for points $(\lambda, \mu)$, where $\lambda=\lambda^{(n)}(\mu)=\lambda^{(m)}(\mu)$ and $n \neq m$. Consider such a point, say $\left(\lambda^{\prime}, \mu^{\prime}\right)$. Theorem 2.2(i) shows that there are exactly two positive integers, say $n$ and $n^{\prime}$, such that $\lambda^{\prime}=\lambda^{(n)}\left(\mu^{\prime}\right)$ and $\lambda^{\prime}=\lambda^{\left(n^{\prime}\right)}\left(\mu^{\prime}\right)$. It is now easy to see that it is possible to pass a smooth curve $h$ through $\left(\lambda^{\prime}, \mu^{\prime}\right)$ such that $h(\mathbf{R}) \cap B\left(\left(\lambda^{\prime}, \mu^{\prime}\right) ; \varepsilon\right) \cap \Sigma_{A}=\left\{\left(\lambda^{\prime}, \mu^{\prime}\right)\right\}$ for $\varepsilon>0$ and sufficiently small. Furthermore, $h$ may be chosen to have the following additional property: namely, if $h\left(t^{\prime}\right)=\left(\lambda^{\prime}, \mu^{\prime}\right)$, then

$$
\operatorname{deg}_{L S}\left(I-h(t) \cdot\left[\begin{array}{ll}
L^{-1} & L^{-1} \\
L^{-1} & L^{-1}
\end{array}\right] ; B(0 ; 1) ; 0\right)
$$

is defined and constant for $t \in\left(t^{\prime}-\delta, t^{\prime}+\delta\right), \delta>0$ and sufficiently small, $t \neq t^{\prime}$. (It is important to note that, while it is possible to choose such an $h$ having both the above properties, not all curves satisfying the first property will satisfy the second. This fact imposes a limitation on the results that follow.)

Before stating our main result on this topic, we need two preliminary results. We begin with the following theorem.

THEOREM 5.1. Let $h$ be a proper crossing of changing degree at $\left(\lambda_{0}, \mu_{0}\right)$ relative to (1.6) as in Definition 4.3. Assume that $h(\mathbf{R}) \cap \Sigma_{A}$ is discrete. Let $C_{\left(\lambda_{0}, \mu_{0}\right)}^{h}$ denote the component of $S \cap(h(\mathbf{R}) \times E)$ meeting $\left(\lambda_{0}, \mu_{0}, 0,0\right)$. Then if $\mathcal{C}_{\left(\lambda_{0}, \mu_{0}\right)}^{h}$ is bounded, the set

$$
\begin{aligned}
& \left\{(\lambda, \mu) \in \Sigma_{A}:(\lambda, \mu, 0,0) \in C_{\left(\lambda_{0}, \mu_{0}\right)}^{h},(\lambda, \mu)=h(t)\right. \text { and } \\
& \lim _{s \rightarrow t^{-}}\left(\operatorname{deg}_{\mathrm{LS}}(I-A(h(s)), B(0 ; 1), 0)\right) \\
& \left.\quad \neq \lim _{s \rightarrow t^{+}}\left(\operatorname{deg}_{\mathrm{LS}}(I-A(h(s)), B(0 ; 1), 0)\right)\right\}
\end{aligned}
$$

contains an even number of elements.

PROOF. The result is readily established by an extension of the methods of [11] to the results of $[\mathbf{7}]$. 
LEMMA 5.2. Let (2.7) hold with $n=1$ and $m=2$. Assume all eigenvalues of $L$ are simple. Let $Z_{0}$ denote the component of $\mathbf{R}^{2} \backslash \Sigma_{A}$ which contains the origin. Let $\left(\lambda_{0}, \mu_{0}\right) \in \Sigma_{A}$ be such that mult $\left(\lambda_{0}, \mu_{0}\right)=1,\left(\lambda^{\prime}, \mu^{\prime}\right) \in\left[\mathbf{R}^{2} \backslash\left(\Sigma_{A} \cup Z_{0}\right)\right] \cup$ $\left\{(\lambda, \mu) \in \Sigma_{A}: \operatorname{mult}(\lambda, \mu)=2\right\}$. Then there is an injective smooth proper crossing of changing degree $h$ at $\left(\lambda_{0}, \mu_{0}\right)$ such that $h\left(t^{\prime}\right)=\left(\lambda^{\prime}, \mu^{\prime}\right)$ for some $t^{\prime}>0$ and such that $h(\mathbf{R}) \cap\left\{(\lambda, \mu) \in \Sigma_{A}\right.$ : $\left.\operatorname{mult}(\lambda, \mu)=1\right\}=\left\{\left(\lambda_{0}, \mu_{0}\right)\right\}$.

PROOF. The result is a consequence of the analysis of $\S 2$. That (2.7) holds with $n=1$ and $m=2$ guarantees that the lower branch of $\lambda^{(1)}$ is the only member of the collection of curves of $\S 2$ with no intersections. The condition that $\left(\lambda^{\prime}, \mu^{\prime}\right) \notin Z_{0}$ is therefore unavoidable for $\left(\lambda_{0}, \mu_{0}\right) \notin \partial Z_{0}$ (the lower branch of $\left.\lambda^{(1)}\right)$. If $\left(\lambda_{0}, \mu_{0}\right) \in$ $\partial Z_{0},\left(\lambda^{\prime}, \mu^{\prime}\right)$ may be taken in the larger set

$$
\left[\mathbf{R}^{2} \backslash \Sigma_{A}\right] \cup\left\{(\lambda, \mu) \in \Sigma_{A}: \operatorname{mult}(\lambda, \mu)=2\right\} .
$$

We now state our main result on this topic.

THEOREM 5.3. Let $\left(\lambda_{0}, \mu_{0}\right)$ and $\left(\lambda^{\prime}, \mu^{\prime}\right)$ be as in Lemma 5.2. Suppose there is a proper crossing of changing degree $h$ at $\left(\lambda_{0}, \mu_{0}\right)$ such that $h(\mathbf{R}) \cap \Sigma_{A}$ is discrete, $\left(\lambda^{\prime}, \mu^{\prime}\right)=h\left(t^{\prime}\right), t^{\prime}>0$, and the set

$$
\begin{aligned}
& \left\{(\lambda, \mu) \in \Sigma_{A} \cap h(\mathbf{R}):(\lambda, \mu)=h(t)\right. \text { where } \\
& \qquad \begin{aligned}
\lim _{s \rightarrow t^{-}} & (\operatorname{deg}(I-A(h(s)), B(0 ; 1), 0)) \\
& \left.\neq \lim _{s \rightarrow t^{+}}\left(\operatorname{deg}_{\mathrm{LS}}(I-A(h(s)), B(0 ; 1), 0)\right)\right\}=\left\{\left(\lambda_{0}, \mu_{0}\right)\right\} .
\end{aligned}
\end{aligned}
$$

Suppose that $\mathrm{C}_{\left(\lambda_{0}, \mu_{0}\right)}^{h}$ is as in Theorem 5.1. Assume also that $C_{\left(\lambda_{0}, \mu_{0}\right)}^{h} \cap[h((-\infty, 0]) \times$ $E]=\left\{\left(\lambda_{0}, \mu_{0}, 0,0\right)\right\}$ and that $\left(\lambda^{\prime}, \mu^{\prime}\right) \notin \Sigma_{A}$. Then one of the following obtains:

(i) There is $t^{*} \in\left(0, t^{\prime}\right)$ such that $C_{\left(\lambda_{0}, \mu_{0}\right)}^{h}$ bifurcates from infinity at $\left(\lambda^{*}, \mu^{*}\right)=$ $h\left(t^{*}\right)$.

(ii) There is $e \neq 0 \in E$, such that $\left(\lambda^{\prime}, \mu^{\prime}, e\right) \in C_{\left(\lambda_{0}, \mu_{0}\right)}^{h}$.

PROOF. Apply Theorem 2.5 of $[\mathbf{7}]$ and Theorem 5.1.

REMARK 5.4. A more general formulation of the result of Theorem 5.3 is possible: If $h$ is a proper crossing of changing degree at $\left(\lambda_{0}, \mu_{0}\right)$ with respect to (1.6) such that $h(\mathbf{R}) \cap \Sigma_{A}$ is discrete with only one change of topological index for (1.8) along $h(\mathbf{R})$, then $C_{\left(\lambda_{0}, \mu_{0}\right)}^{h}$ satisfies alternative (i) of Theorem 4.4. However, Theorem 5.3 amply demonstrates the impact that the structure of $\Sigma_{A}$ has on the process of identifying global behavior of bifurcating nontrivial solutions to problems of general type (3.1). We note that no differentiability requirements are made of such problems, beyond those of (1.4). Thus the methods described here are applicable for more general nonlinearities than those of (1.6).

COROllary 5.5. Suppose $\left(\lambda_{0}, \mu_{0}\right),\left(\lambda^{\prime}, \mu^{\prime}\right)$ and $h$ are as in Theorem 5.3 and that (ii) obtains. Then if $\left(\lambda^{\prime}, \mu^{\prime}, e\right) \in S_{\left(\lambda_{0}, \mu_{0}\right)}^{h}$ (see $\left.\S 4\right)$, there is a set $T_{\left(\lambda^{\prime}, \mu^{\prime}, e\right)}$ of dimension $\geq 2$ such that $\left(\lambda^{\prime}, \mu^{\prime}, e\right) \in T_{\left(\lambda^{\prime}, \mu^{\prime}, e\right)} \subseteq S_{\left(\lambda_{0}, \mu_{0}\right)}$.

REMARK 5.6. Note that it is not possible to have $h(t)=\{(t, 0): t \in \mathbf{R}\}$ or $h(t)=\{(0, t): t \in \mathbf{R}\}$. Consequently, under the assumption that the eigenvalues of 
$L$ are all simple, a result distinguishing global Rabinowitz alternatives purely on the basis of the linearization of the problem as in Theorem 5.3 is not available in the single equation case. Additional information on the qualitative behavior such as global preservation of nodal structure to solutions in the case of nonlinear SturmLiouville boundary value problems is needed. That such is the case is indicative of the extra information obtainable in several parameter systems of equations. This information should be exploitable in much more general contexts than we have pursued in this section.

6. Global persistence of positive solutions. The descriptions of nontrivial bifurcating solutions to (1.6) provided by Theorem 3.3 are local. Some global results of this type may be realized as follows. Consider once again (1.6). Assume that $L$ (or more generally, $A$ ) is such that strong maximum principles [17] apply, $L$ has simple eigenvalues and, as usual, that $(2.5)-(2.6)$ is valid. We make the following additional assumptions on nonlinearities $\tilde{f}, \tilde{g}$ :

$$
\tilde{f} \text { and } \tilde{g} \operatorname{map}[0, \infty) \times[0, \infty) \text { into }[0, \infty) ;
$$

$\tilde{f}$ and $\tilde{g}$ are odd.

Lemma 6.1. Suppose (6.1) holds and let $D$ be as in Theorem 3.3. Suppose that $\left\{\left(\lambda_{n}, \mu_{n}, u_{n}, v_{n}\right)\right\}_{n=1}^{\infty} \subseteq S$ is a sequence with $\lambda_{n}>0, \mu_{n}>0, u_{n} \in D, v_{n} \in D$ for $n \geq 1$. Then if $\left(\lambda_{n}, \mu_{n}, u_{n}, v_{n}\right) \rightarrow\left(\lambda_{0}, \mu_{0}, u_{0}, v_{0}\right)$ as $n \rightarrow \infty$, where $\lambda_{0}>0$ and $\mu_{0}>0$, either $\left(u_{0}, v_{0}\right) \in D \times D$ or $u_{0} \equiv 0$ and $v_{0} \equiv 0$.

Proof. $u_{0} \geq 0$ and $v_{0} \geq 0$. Suppose $\left(u_{0}, v_{0}\right) \neq(0,0)$ and $\left(u_{0}, v_{0}\right) \notin D \times D$. Then one of the following holds:

(i) There is $x \in \Omega$ such that $u_{0}(x)=0$ or $v_{0}(x)=0$.

(ii) There is $\eta \in \partial \Omega$ such that $\left(\partial u_{0} / \partial \nu\right)(\eta)=0$ or $\left(\partial v_{0} / \partial \nu\right)(\eta)=0$.

If (i) holds, suppose with no loss of generality that $u_{0}(x)=0$. Then $L u_{0}$ (or $\left.A u_{0}\right)=\lambda_{0} f_{1} u_{0}+\lambda_{0} f_{2} v_{0}+\lambda_{0} \tilde{f}\left(u_{0}, v_{0}\right) \geq 0$ on $\Omega$, by (2.5)-(2.6) and (6.1), and $u_{0} \geq 0$ on $\bar{\Omega}$. Since $u_{0}(x)=0, u_{0} \equiv 0$ by the maximum principle. Hence $\lambda_{0} f_{2} v_{0}+$ $\lambda_{0} \tilde{f}\left(0, v_{0}\right) \equiv 0$. By $(6.1)$ and (2.5)-(2.6), $v_{0} \equiv 0$, a contradiction. If (ii) holds and $\left(\partial u_{0} / \partial \nu\right)(\eta)=0$, then $L u_{0}$ (or $\left.A u_{0}\right) \geq 0$ on $\Omega, u_{0} \geq 0$ on $\partial \Omega, u_{0}(\eta)=0$ and $\left(\partial u_{0} / \partial \nu\right)(\eta)=0$. Thus $u_{0} \equiv 0$ by the strong maximum principle, and $v_{0} \equiv 0$ as above, a contradiction. Cf. [23].

Lemma 6.2. Suppose $(\lambda, \mu) \in \Sigma_{A}$ such that $(\lambda, \mu)$ satisfies both

$$
\lambda=\frac{\lambda_{m}\left(g_{2} \mu-\lambda_{m}\right)}{\left(f_{1} g_{2}-f_{2} g_{1}\right)-\lambda_{m} f_{1}}
$$

and

$$
\lambda=\frac{\lambda_{n}\left(g_{2} \mu-\lambda_{n}\right)}{\left(f_{1} g_{2}-f_{2} g_{1}\right) \mu-\lambda_{n} f_{1}},
$$

$n<m$. Suppose also that the eigenvalues of $L$ are all simple. Then if $(\lambda, \mu, \bar{u}, \bar{v})$ is a solution of $(1.7)$ with $(\bar{u}, \bar{v}) \neq(0,0)$, then there is $x_{0} \in \Omega$ such that $\bar{u}\left(x_{0}\right)<0$ or $\bar{v}\left(x_{0}\right)<0$.

Proof. Since $(\lambda, \mu)$ satisfies (6.3) and (6.4) with $n<m$, then $m>1$ and $\mu>$ $\lambda_{n} f_{1} /\left(f_{1} g_{2}-f_{2} g_{1}\right)$. Furthermore, $c(\lambda, \mu)+d(\lambda, \mu)=\lambda_{m}$ and $c(\lambda, \mu)-d(\lambda, \mu)=\lambda_{n}$. 
Arguing as in Proposition 2.3 and using the simplicity of $\lambda_{n}$ and $\lambda_{m}$, it follows that

$$
\begin{aligned}
\{(x, y) & \in E:(\lambda, \mu, x, y) \text { satisfies }(1.7)\} \\
& =\left\{c_{1}\left(\begin{array}{c}
1 \\
\beta_{m}
\end{array}\right) x_{m}+c_{2}\left(\begin{array}{c}
1 \\
\beta_{n}
\end{array}\right) x_{n}: c_{1}, c_{2} \in \mathbf{R}\right\},
\end{aligned}
$$

where $L x_{m}=\lambda_{m} x_{m}, L x_{n}=\lambda_{n} x_{n}, \beta_{m}>0$ and $\beta_{n}<0$. Since $m>1, x_{m}$ necessarily changes sign on $\Omega$. In the special case $n=1, x_{1}$ may be chosen positive on $\Omega$. In this instance, as $\mu>\lambda_{1} f_{1} /\left(f_{1} g_{2}-f_{2} g_{1}\right)$ and $\beta_{1}<0, \beta_{1} x_{1}<0$ on $\Omega$. Hence it suffices to verify the claim only in case $c_{1} c_{2} \neq 0$. By Theorem 1 of $[\mathbf{1}]$, there is an open subset $\Omega^{\prime}$ of $\Omega$ such that $x_{n}>0$ on $\Omega^{\prime}$ but $x_{m}$ changes sign in $\Omega^{\prime}$. There are four cases: $c_{1}>0, c_{2}>0 ; c_{1}>0, c_{2}<0 ; c_{1}<0, c_{2}>0 ; c_{1}<0, c_{2}<0$. We argue only in case $c_{1}>0$ and $c_{2}>0$. In this case there is $x_{0} \in \Omega^{\prime}$ such that $x_{m}\left(x_{0}\right)<0$. Then $\bar{v}=c_{1} \beta_{m} x_{m}+c_{2} \beta_{n} x_{n}$ is such that $\bar{v}\left(x_{0}\right)<0$.

We may now give the following result.

THEOREM 6.3. Suppose $L$ is such that strong maximum principles apply and that $L$ has simple eigenvalues $\lambda_{1}<\lambda_{2}<\cdots<\lambda_{n} \rightarrow \infty$. Suppose $f$ and $g$ satisfy (6.1)-(6.2). Let $C$ be a continuum in $S \cap((0, \infty) \times(0, \infty) \times E)$ such that $C$ meets $\Sigma_{A}^{1} \times\{0\}$, where

$$
\Sigma_{A}^{1}=\left\{(\lambda, \mu) \in \Sigma_{A}: \lambda=\frac{\lambda_{1}\left(g_{2} \mu-\lambda_{1}\right)}{\left(f_{1} g_{2}-f_{2} g_{1}\right) \mu-\lambda_{1} f_{1}}, 0<\mu<\frac{\lambda_{1}}{g_{2}}\right\} .
$$

Then

$$
\begin{aligned}
C \backslash\left(\Sigma_{A}^{1} \times\{0\}\right) & \subseteq[(0, \infty) \times(0, \infty) \times D \times D] \\
& \cup[(0, \infty) \times(0, \infty) \times(-D) \times(-D)] .
\end{aligned}
$$

PROOF. Theorems 2.5 and 3.2 and Lemma 6.1 show that the result holds as long as $C \cap\left(\mathbf{R}^{2} \times\{0\}\right) \subseteq\left(\Sigma_{A}^{1}\right) \times\{0\}$. That such is the case follows from Proposition 2.3 and Lemma 6.2.

REMARK 6.4. It remains unresolved whether conditions similar to (6.1) exist so that analogous results obtain for higher nodal properties in case $\bar{\Omega}=[a, b]$.

REMARK 6.5. Theorem 6.3 may also be obtained by adapting ordered Banach space methods (e.g. [2]) to a multiparameter situation.

7. The $n$-parameter case. Now consider (1.3). The linearization of (1.3) at $\left(u_{1}, \ldots, u_{n}\right)=(0, \ldots, 0)$ is given by the system of equations

$$
L v_{i}(x)=\lambda_{i} \sum_{j=1}^{n} f_{i j} v_{j}(x)
$$

$i=1, \ldots, n$, where $x \in \Omega$ and $v_{i}(x) \equiv 0$ for $x \in \partial \Omega, i=1, \ldots, n$. The coefficient $f_{i j}=\left(\partial f_{i} / \partial t_{j}\right)(0, \ldots, 0)$, where $f_{i}=f_{i}\left(t_{1}, \ldots, t_{n}\right), t_{k} \in \mathbf{R}, k=1, \ldots, n$. If $n=2$, (7.1) reduces to (1.7). In analogy to $(2.3)$, if (7.1) holds, then

$$
\operatorname{det}\left[\begin{array}{cc}
L-\lambda_{1} f_{11} & -\lambda_{1} f_{12} \cdots-\lambda_{1} f_{1 n} \\
-\lambda_{2} f_{21} & L-\lambda_{2} f_{22} \cdots-\lambda_{2} f_{2 n} \\
\vdots & \vdots \\
-\lambda_{n} f_{n 1} & -\lambda_{n} f_{n 2} \cdots L-\lambda_{n} f_{n n}
\end{array}\right] w=0
$$


with $w=v_{i}, i=1, \ldots, n$. Observe that if $A(L)$ denotes the operator (a polynomial in terms of $L$ ) on the left-hand side of (7.2), then

$$
A(L)=\prod_{i=1}^{n}\left(L-\phi_{i}\left(\lambda_{1}, \ldots, \lambda_{n}\right)\right)
$$

where $\phi_{i}\left(\lambda_{1}, \ldots, \lambda_{n}\right)$ is an algebraic expression in $\lambda_{1}, \ldots, \lambda_{n}, i=1, \ldots, n$. Let $\mu_{1}<\mu_{2}<\cdots<\mu_{m}<\cdots$ denote the eigenvalues $L$ subject to zero boundary data. Then $\Sigma_{A}=\left\{\left(\lambda_{1}, \ldots, \lambda_{n}\right) \in \mathbf{R}^{n}: \phi_{i}\left(\lambda_{1}, \ldots, \lambda_{n}\right)=\mu_{m}\right.$ for some $i \in\{1, \ldots, n\}$ and for some $\left.m \in Z^{+}\right\}$. A detailed analysis of the bifurcation phenomena associated with (1.3) in the spirit of $\S \S 3,4,5$ and 6 is possible once a detailed examination of $\Sigma_{A}$ is made as in $\S 2$.

8. An illustration. Now consider an application of the results of $\S \S 2-6$ to a particular example. Let us take $\bar{\Omega}=[0, \pi]$ and examine

$$
\begin{aligned}
& -u^{\prime \prime}(x)=\lambda\left[2 u(x)+v(x)+u^{2}(x) v(x)+u^{3}(x)\right], \\
& -v^{\prime \prime}(x)=\mu\left[u(x)+v(x)+u^{2}(x) v(x)+v^{3}(x)\right]
\end{aligned}
$$

$u(0)=0=u(\pi), v(0)=0=v(\pi)$.

The problem

$$
-w^{\prime \prime}(x)=\alpha w(x)
$$

$w(0)=0=w(\pi)$, has simple eigenvalues $\alpha=m^{2}, m=1,2, \ldots$, with corresponding eigenfunctions $\sin m t$. Furthermore in terms of $\S 2, f_{1}=2, f_{2}=1, g_{1}=1, g_{2}=1$ and $f_{1} g_{2}-f_{2} g_{1}=1$. Thus a simple computation shows

$$
\Sigma_{A}=\left\{(\lambda, \mu) \in \mathbf{R}^{2}: \lambda=\frac{n^{2}\left(\mu-n^{2}\right)}{\mu-2 n^{2}} \text { for some } \mu \in \mathbf{R} \text { and } n \in Z^{+}\right\} .
$$

Let $\lambda^{(n)}$ denote the linear fractional transformation given by

$$
\lambda^{(n)}(\mu)=\frac{n^{2}\left(\mu-n^{2}\right)}{\mu-2 n^{2}} .
$$

Condition (2.7) for the intersection of $\lambda^{(n)}$ and $\lambda^{(m)}, n<m$, specialized to (8.1), becomes

$$
n^{2} / m^{2} \leq 3-2 \sqrt{2}
$$

Furthermore, Theorem 2.5 implies that if $\lambda^{(n)}(\mu) \neq \lambda^{(m)}(\mu)$ for any $m \neq n$,

$$
\operatorname{mult}\left(\lambda^{(n)}(\mu), \mu\right)=1 \text {. }
$$

One readily observes that $\lambda^{(1)}$ does not meet $\lambda^{(2)}$ but that $\lambda^{(1)}$ does meet $\lambda^{(k)}$ for $k \geq 3$. Figure 2 lists the linear fractional transformation $\lambda^{(k(n))}, k(n)>n$, of first intersection for $\lambda^{(n)}, n \leq 15$, and Figure 3 gives a schematic diagram of the intersections of $\lambda^{(n)}, n \leq 8$. 


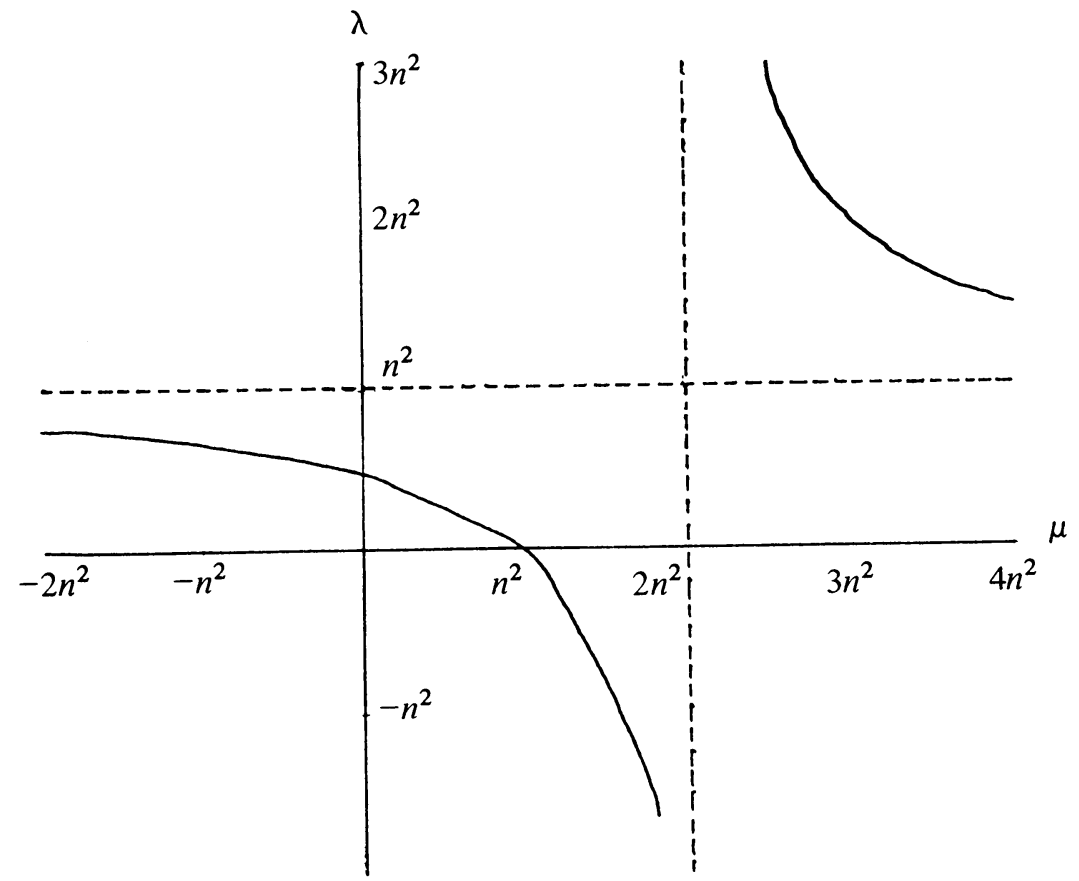

FIGURE 1

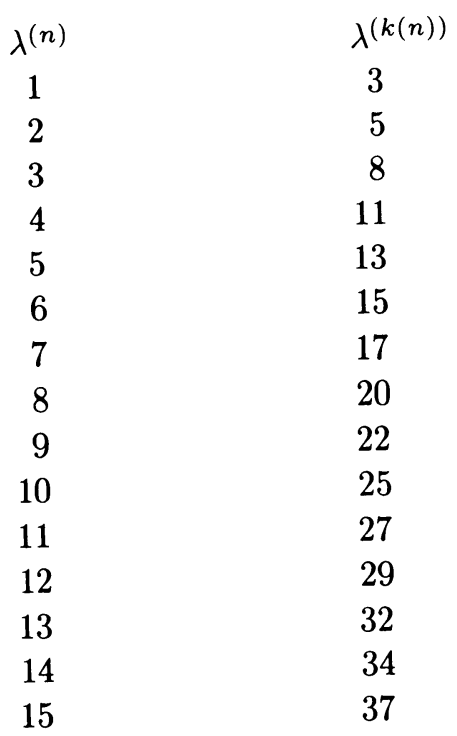

FIGURE 2 
Let

$$
\Sigma_{A}^{1 l}=\left\{(\lambda, \mu): \lambda=\frac{\mu-1}{\mu-2},-\infty<\mu<2\right\}
$$

and let $T^{1 l}$ denote the component of $\mathbf{R}^{2} \backslash \Sigma_{A}^{1 l}$ containing the point $\{(\lambda, \mu)\}=\{(1,0)\}$ (see Figure 3). Then the analysis of $\S 5$ holds for parameter values in $T^{1 l}$. In particular, Lemma 5.2 is valid for $\left(\lambda_{0}, \mu_{0}\right) \in \tilde{\Sigma}_{A}=\Sigma_{A} \backslash \Sigma_{A}^{1 l}$ and $\left(\lambda^{\prime}, \mu^{\prime}\right) \in T^{1 l} \backslash \tilde{\Sigma}_{A}$.

Observe also that (8.1) is such that (6.1)-(6.2) are satisfied. Since the eigenvalues of (8.2) are all simple, we can conclude from $\S 6$ that solutions of the form $(\lambda, \mu, u, v)$ with $u(x)>0$ on $(0, \pi)$ and $v(x)>0$ on $(0, \pi)$ emanate from $\Sigma_{A}^{1 l} \cap[(0, \infty) \times(0, \infty)]$ and persist globally for $\lambda>0, \mu>0$.

It remains to observe that $r=s=3$ in the statement of Theorem 3.6 for all simple bifurcation points (except possibly when $(\lambda, \mu)=\left(\frac{1}{2} n^{2}, 0\right)$ or $\left.\left(0, n^{2}\right)\right)$. To this end, first observe if $(w, z)=(\alpha \sin n t, \beta \sin n t)$ is a solution to the linearization of $(8.1)$ at $(\lambda, \mu)=\left(n^{2}\left(\mu-n^{2}\right) /\left(\mu-2 n^{2}\right), \mu\right)$, then $\beta\left(n^{2}-\mu\right)=\mu \alpha$. Hence if $u \neq n^{2}$,

$$
\beta=\alpha \mu /\left(n^{2}-\mu\right) \text {. }
$$

Next observe that if $1 / \lambda(\mu)+[\varepsilon(\mu)]^{2} / \mu \neq 0$, where $\lambda(\mu)=n^{2}\left(\mu-n^{2}\right) /\left(\mu-2 n^{2}\right)$ and $\varepsilon(\mu)=\mu /\left(n^{2}-\mu\right)$, then the quantity

$$
\int_{0}^{\pi}\left(\frac{w^{2}}{\lambda(\mu)}+\frac{z^{2}}{\mu}\right) d x \neq 0
$$

and the desired result is obtained by calculating that (3.15) is nonzero. Note that

$$
\begin{aligned}
\frac{1}{\lambda(\mu)} & +\frac{[\varepsilon(\mu)]^{2}}{\mu}=\frac{\mu-2 n^{2}}{n^{2}\left(\mu-n^{2}\right)}+\frac{1}{\mu}\left[\frac{\mu^{2}}{\left(n^{2}-\mu\right)^{2}}\right] \\
& =\frac{\left(\mu-2 n^{2}\right)\left(\mu-n^{2}\right)+n^{2} \mu}{n^{2}\left(\mu-n^{2}\right)^{2}}=\frac{\left(\mu-n^{2}\right)^{2}+n^{4}}{n^{2}\left(\mu-n^{2}\right)^{2}} \neq 0 \text { if } \mu \neq 0, n^{2}, 2 n^{2} .
\end{aligned}
$$

In this example, one may readily conclude that (3.15) being nonzero is equivalent to

$$
\sum_{l+m=3}[\varepsilon(\mu)]^{m}\left[\left(g_{2} f_{l m}-f_{2} g_{l m}\right)+\frac{\lambda(\mu)}{\mu} \varepsilon(\mu)\left(f_{1} g_{l m}-f_{2} f_{l m}\right)\right] \neq 0,
$$

where $\varepsilon(\mu), \lambda(\mu)$ are as above, and

$$
\begin{array}{ll}
f_{03}=0, & g_{03}=1 \\
f_{12}=0, & g_{12}=0 \\
f_{21}=1, & g_{21}=1 \\
f_{30}=1, & g_{30}=0 .
\end{array}
$$

Calculation reveals $(8.6)$ to be

$$
\begin{aligned}
& 2\left(\frac{n^{2}\left(\mu-n^{2}\right)}{\mu\left(\mu-2 n^{2}\right)}\right)\left(\frac{\mu}{n^{2}-\mu}\right)^{4}-\left(\frac{\mu}{n^{2}-\mu}\right)^{3}+\left(\frac{n^{2}\left(\mu-n^{2}\right)}{\mu\left(\mu-2 n^{2}\right)}\right)\left(\frac{\mu}{n^{2}-\mu}\right)^{2} \\
& \quad-\left(\frac{n^{2}\left(\mu-n^{2}\right)}{\mu\left(\mu-2 n^{2}\right)}\right)\left(\frac{\mu}{n^{2}-\mu}\right)+1
\end{aligned}
$$

(8.7) simplifies to

$$
\frac{-2 \mu^{4}+3 n^{2} \mu^{3}-4 n^{4} \mu^{2}+3 n^{6} \mu-n^{8}}{\left(\mu-2 n^{2}\right)\left(n^{2}-\mu\right)^{3}} .
$$




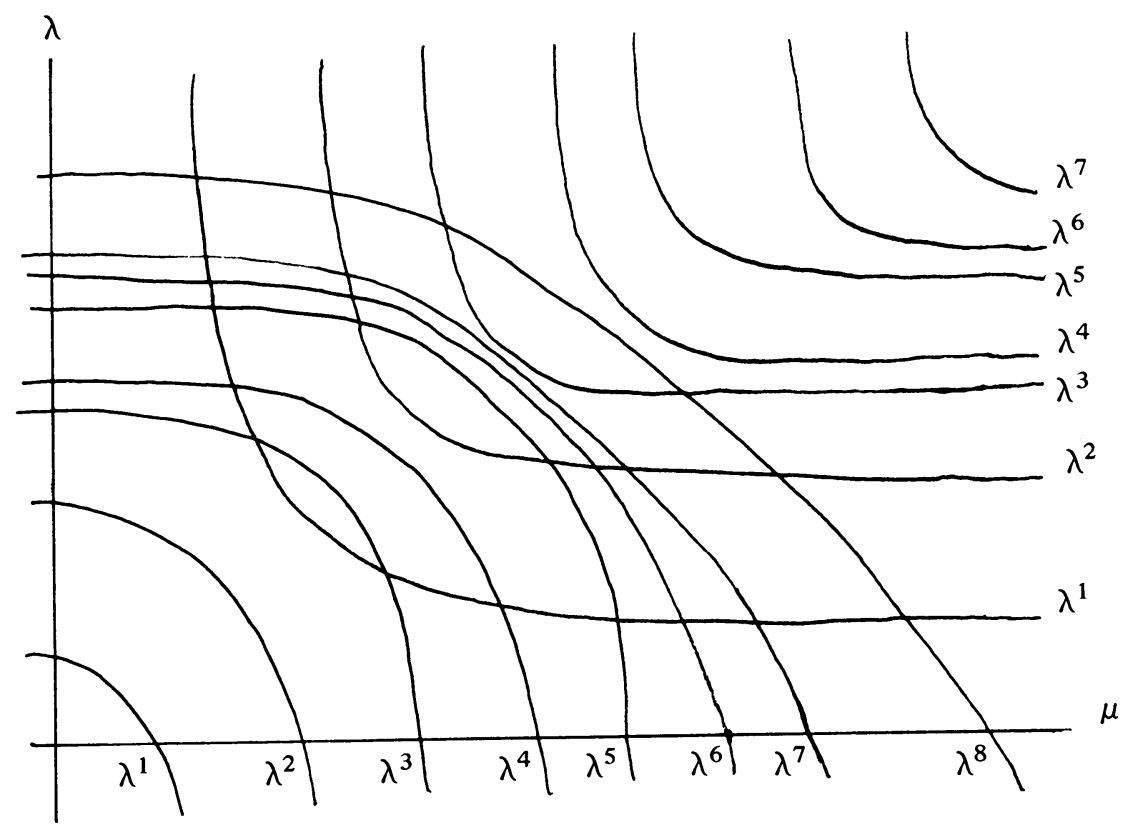

FIGURE 3

To show $(8.8) \neq 0$ if $\mu \neq 0, n^{2}$, or $2 n^{2}$, it suffices to consider the polynomial $P(n, \mu)$ given by

$$
P(n, \mu)=-2 \mu^{4}+3 n^{2} \mu^{3}-4 n^{4} \mu^{2}+3 n^{6} \mu-n^{8} .
$$

Let $H(n, \mu)=-2 \mu^{4}+3 n^{2} \mu^{3}$ and $G(n, \mu)=-4 n^{4} \mu^{2}+3 n^{6} \mu$. A simple computation shows that $P^{\prime \prime}(n, \mu)<0$ for all $\mu \in \mathbf{R}$ and that $P^{\prime}\left(n, \frac{1}{2} n^{2}\right)=\frac{1}{4} n^{6}$ while $P^{\prime}\left(n, \frac{3}{5} n^{2}\right)=\frac{-36}{125} n^{6}$, where ${ }^{\prime}=d / d \mu$. Consequently,

$$
\begin{aligned}
\max _{\mu \in \mathbf{R}} P(n, \mu) & \leq \max _{\mu \in\left[\frac{1}{2} n^{2}, \frac{3}{5} n^{2}\right]} P(n, \mu) \leq \max _{\mu \in\left[\frac{1}{2} n^{2}, \frac{3}{5} n^{2}\right]} H(n, \mu) \\
& +\max _{\mu \in\left[\frac{1}{2} n^{2}, \frac{3}{5} n^{2}\right]} G(n, \mu)-n^{8} .
\end{aligned}
$$

Now $H^{\prime}(n, \mu)=-8 \mu^{3}+9 n^{2} \mu^{2}, H^{\prime \prime}(n, \mu)=-24 \mu^{2}+18 n^{2} \mu, G^{\prime}(n, \mu)=-8 n^{4} \mu+$ $3 n^{6}$, and $G^{\prime \prime}(n, \mu)=-8 n^{4}$. It is now easy to see that

$$
\max _{\mu \in\left[\frac{1}{2} n^{2}, \frac{3}{5} n^{2}\right]} H(n, \mu)=H\left(n, \frac{3}{5} n^{2}\right)=\frac{243}{625} n^{8} .
$$

and that

$$
\max _{\mu \in\left[\frac{1}{2} n^{2}, \frac{3}{5} n^{2}\right]} G(n, \mu)=G\left(n, \frac{1}{2} n^{2}\right)=\frac{1}{2} n^{8} .
$$

Thus

$$
P(n, \mu) \leq \max _{\mu \in\left[\frac{1}{2} n^{2}, \frac{3}{5} n^{2}\right]} P(n, \mu) \leq\left(\frac{243}{625}+\frac{1}{2}-1\right) n^{8}<0 .
$$

Thus $(8.7) \neq 0$, and we conclude the existence of two "small" nontrivial solutions near $\left(\lambda^{(n)}(\mu), \mu, 0,0\right)$, where $\mu \cdot \lambda^{(n)}(\mu) \neq 0$ and $\operatorname{mult}\left(\lambda^{(n)}(\mu), \mu\right)=1$. 


\section{BIBLIOGRAPHY}

1. S. Ahmad and A. C. Lazer, On the role of Hopf's maximum principle in elliptic Sturmiun theory, Houston J. Math. 5 (1979), 155-158.

2. H. Amann, Nonlinear operators in ordered Banach spaces and some applications to nonlinear boundary value problems, Nonlinear Operators and Calculus of Variations (Bruxelles 1975), Lecture Notes in Math., Vol. 543, Springer, Berlin and New York, 1976, pp. 1-55.

3. J. C. Alexander and S. S. Antman, Global and local behavior of bifurcating multidimensional continua of solutions for multiparameter nonlinear eigenvalue problems, Arch. Rational Mech. Anal. 76 (1981), 339-354.

4. _ Global behavior of solutions of nonlinear equations depending on infinitedimensional parameters, Indiana Univ. Math. J. 32 (1983), 39-62.

5. P. J. Browne and B. D. Sleeman, Non-linear multiparameter eigenvalue problems for ordinary differential equations, J. Math. Anal. Appl. 77 (1980), 425-432.

6. _ Nonlinear multiparameter Sturm-Liouville problems, J. Differential Equations 34 (1979), 139-146.

7. R. S. Cantrell, $A$ homogeneity condition guaranteeing bifurcation in multiparameter nonlinear eigenvalue problems, Nonlinear Anal. 8 (1984), 159-169.

8. __ Multiparameter bifurcation problems and topological degree, J. Differential Equations 52 (1984), 39-51.

9. __ Multiparameter bifurcation problems for second order ordinary differential equations, Rocky Mountain J. Math. 12 (1982), 795-806.

10. M. G. Crandall and P. H. Rabinowitz, Nonlinear Sturm-Liouville eigenvalue problems and topological degree, J. Math. Mech. 19 (1970), 1083-1102.

11. E. N. Dancer, On the structure of solutions of non-linear eigenvalue problems, Indiana Univ. Math. J. 23 (1974), 1069-1076.

12. J. Dieudonné, Foundations of modern analysis, Academic Press, New York, 1960.

13. M. Fairman, Ph.D. Thesis, Univ. of Toronto, 1966.

14. P. M. Fitzpatrick, I. Massabo and J. Pejsachowicz, Complementing maps, continuation and global bifurcation, Bull. Amer. Math. Soc. (N.S.) 9 (1983), 79-81.

15. __ Global several-parameter bifurcation and continuation theorems: a unified approach via complementing maps, Math. Ann. 263 (1983), 61-73.

16. On the covering dimension of the set of solutions of some nonlinear equations, Trans. Amer. Math. Soc. (to appear).

17. D. Gilbarg and N. S. Trudinger, Elliptic partial differential equations of the second order, Grundlehren der Math. Wissenschaften, Vol. 222, Springer-Verlag, Berlin, 1977.

18. M. Golubitsky and V. Guillemin, Stable mappings and their singularities, Springer-Verlag, New York, 1973.

19. E. L. Ince, Ordinary differential equations, Longmans, Greene, and Co., London, 1927.

20. M. A. Krasnosel'skii, Topological methods in the theory of nonlinear integral equations, Macmillan, New York, 1965.

21. P. L. Lions, On the existence of positive solutions of semilinear elliptic equations, SIAM Rev. 24 (1982), 441-467.

22. M. H. Protter, The generalized spectrum of second order elliptic systems, Rocky Mountain J. Math. 9 (1979), 503-518.

23. P. H. Rabinowitz, Some aspects of nonlinear eigenvalue problems, Rocky Mountain J. Math. 3 (1973), 161-202.

24. 487-513.

25. R. E. L. Turner, Nonlinear Sturm-Liouville problems, J. Differential Equations 10 (1971), 141-146.

26. D. W. Zachmann, Multiple solutions of coupled Sturm-Liouville systems, J. Math. Anal. Appl. 54 (1976), 467-475.

Department of Mathematics and Computer Science, The University of Miami, Coral Gables, Florida 33124 